\title{
Loss of Tribbles pseudokinase-3 promotes Akt-driven tumorigenesis via FOXO inactivation
}

\author{
M Salazar ${ }^{1,2,14,15}$, M Lorente ${ }^{1,2,14}$, E García-Taboada ${ }^{1}$, E Pérez Gómez ${ }^{1,3}$, D Dávila, ${ }^{1,2}$, P Zúñiga-García ${ }^{4}$, J María Flores ${ }^{5}$, A Rodríguez ${ }^{5}$,

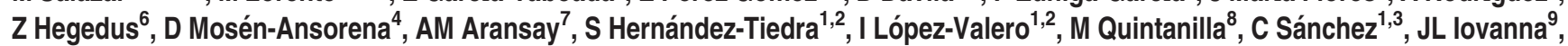 \\ N Dusetti ${ }^{9}$, M Guzmán $^{1,10}$, SE Francis ${ }^{11}$, A Carracedo ${ }^{4,12,13}$, E Kiss-Toth ${ }^{11}$ and G Velasco*,1,2
}

Tribbles pseudokinase-3 (TRIB3) has been proposed to act as an inhibitor of AKT although the precise molecular basis of this activity and whether the loss of TRIB3 contributes to cancer initiation and progression remain to be clarified. In this study, by using a wide array of in vitro and in vivo approaches, including a Trib3 knockout mouse, we demonstrate that TRIB3 has a tumorsuppressing role. We also find that the mechanism by which TRIB3 loss enhances tumorigenesis relies on the dysregulation of the phosphorylation of AKT by the mTORC2 complex, which leads to an enhanced phosphorylation of AKT on Ser473 and the subsequent hyperphosphorylation and inactivation of the transcription factor FOXO3. These observations support the notion that loss of TRIB3 is associated with a more aggressive phenotype in various types of tumors by enhancing the activity of the mTORC2/AKT/FOXO axis.

Cell Death and Differentiation (2015) 22, 131-144; doi:10.1038/cdd.2014.133; published online 29 August 2014

Pseudokinases constitute a group of proteins that have a kinase-like domain that lacks at least one of the conserved catalytic residues. ${ }^{1,2}$ Different studies have shown that some pseudokinases can exhibit low levels of kinase activity, while others have critical roles as activators of their specific targets. $^{1,2}$ Moreover, aberrant regulation of pseudokinases has been implicated in the etiology and progression of a variety of diseases, including cancer. ${ }^{3}$

The Tribbles family of pseudokinases was first described in Drosophila as a negative regulator of cell division in early embryogenesis. ${ }^{4-7}$ There are three mammalian Tribbles isoforms (Trib1, Trib2 and Trib3), homologs to the Drosophila tribbles proteins, and they all share a highly conserved central kinase-like domain, which lacks catalytic residues, and a 'tribbles specific' C-terminal domain, which has been proposed to participate in the binding to different Tribbles partners. ${ }^{8}$

Tribbles pseudokinase-3 (TRIB3; also named TRB3, NIPK and SKIP3) has been proposed to interact with several proteins, including the transcription factors activating transcription factor 4 (ATF-4) and $\mathrm{CHOP}^{9}$ as well as with several MAPKs. ${ }^{10}$ TRIB3 has also been shown to interact and inhibit
AKT, ${ }^{11}$ which has been suggested to suppress insulin signaling. ${ }^{11,12}$ In addition, administration of different anticancer agents promotes cancer cell death via TRIB3 upregulation and the subsequent inhibition of Akt. ${ }^{13-19}$ However, the precise molecular basis of the regulation of Akt by TRIB3 and whether loss of this pseudokinase may contribute to cancer initiation and progression remains to be clarified.

In this study, we investigated the effect of the genetic inactivation of TRIB3 in several cellular and animal models of cancer. Our findings indicate that genetic inhibition of TRIB3 enhances tumorigenesis and that this effect is due - at least primarily - to a selective inactivation of the transcription factor FOXO by the mammalian target of rapamycin complex 2 (mTORC2)/AKT axis.

\section{Results}

Genetic inhibition of TRIB3 facilitates oncogene transformation and enhances the tumorigenicity of cancer cells. As a first approach to analyze the role of TRIB3 in cancer generation and progression, we investigated the

\footnotetext{
${ }^{1}$ Department of Biochemistry and Molecular Biology I, School of Biology, Complutense University, Madrid, Spain; ${ }^{2}$ Instituto de Investigaciones Sanitarias San Carlos (IdISSC), Madrid, Spain; ${ }^{3}$ Instituto de Investigación Hospital 12 de Octubre (I+12), Madrid, Spain; ${ }^{4} \mathrm{CIC}$ bioGUNE, Bizkaia Technology Park, Derio, Spain; ${ }^{5}$ Department of Animal Surgery and Medicine, School of Veterinary, Complutense University, Madrid, Spain; ${ }^{6}$ Institute of Biophysics, Hungarian Academy of Sciences, Szeged, Hungary; ${ }^{7} \mathrm{CIC}$ bioGUNE-CIBERehd, Bizkaia Technology Park, Derio, Spain; ${ }^{8}$ Instituto de Investigaciones Biomédicas Alberto Sols, Consejo Superior de Investigaciones Científicas-Universidad Autónoma de Madrid (CSIC-UAM), Madrid, Spain; ${ }^{9}$ Centre de Recherche en Carcérologie de Marseille (CRCM), INSERM UMR, CNRS UMR 7258, Aix Marseille Université and Institut Paoli Calmette, Marseille, France; ${ }^{10}$ Centro de Investigación Biomédica en Red sobre Enfermedades Neurodegenerativas (CIBERNED) and Instituto Ramón y Cajal de Investigaciones Sanitarias (IRYCIS), Madrid, Spain; ${ }^{11}$ Department of Cardiovascular Science, University of Sheffield, Sheffield, UK; ${ }^{12}$ Ikerbasque, Basque Foundation for Science, Bilbao, Spain and ${ }^{13}$ Biochemistry and Molecular Biology Department, University of the Basque Country (UPV/EHU), Bilbao, Spain

*Corresponding author: G Velasco, Department of Biochemistry and Molecular Biology I, School of Biology, Complutense University, Calle José Antonio Nováis 2 , Madrid 28040, Spain. Tel: + 3491 3944668; Fax: + 3491 3944672; E-mail: gvd@ bbm1.ucm.es

${ }^{14}$ These authors contributed equally to this work.

${ }^{15}$ Current address: Cell Division and Cancer Group, Spanish National Cancer Research Centre (CNIO), Madrid E-28029, Spain.

Abbreviations: ATF-4, activating transcription factor 4; BAD, BCL2-associated agonist of cell death; BIM, BCL2-like 11; DMBA, 7,12-dimethylbenz[a]anthracene; FOXO3, Forkhead box O3; GSK3, glycogen synthase kinase 3; MEF, mouse embryonic fibroblast; mTORC2, mammalian target of rapamycin complex 2; PRAS40, proline-rich AKT substrate; PTEN, phosphatase and tensin homolog; RICTOR, rapamycin-insensitive companion of mTOR; TPA, 12-0-tetradecanoylphorbol-13acetate; TRIB3, Tribbles pseudokinase-3

Received 06.2.14; revised 11.7.14; accepted 24.7.14; Edited by S Fulda; published online 29.8.14
} 
susceptibility to transformation of mouse embryonic fibroblasts (MEFs) derived from Trib3 ${ }^{-1}$ and wild-type (WT) animals (see Supplementary Methods section and Supplementary Figures S1a-e). Expression of Ras ${ }^{\mathrm{V} 12}$ (a constitutively active form of the oncogene Ras) was capable of transforming MEFs derived from Trib3-deficient mice (Figure 1a). Similar results were obtained when the SV40 T-large antigen was used (Supplementary Figure S1f), suggesting that loss of TRIB3 facilitates oncogene-induced transformation overall. In line with this idea, a higher number of Trib3-deficient Ras ${ }^{\mathrm{V} 12}$-transformed (Figure 1b) and SV40-T large antigen-transformed (Supplementary Figure S1g) MEFs were obtained after several days in culture when compared with their corresponding WT counterparts.

Next, we generated Ras ${ }^{\mathrm{V} 12} / \mathrm{E} 1 \mathrm{~A}$-transformed MEFs as a cellular model to conduct additional mechanistic studies on the role of TRIB3 in cancer. In line with the aforementioned results, a higher percentage of $\mathrm{Ras}^{\mathrm{V} 12} / \mathrm{E} 1 \mathrm{~A}$-transformed Trib3-deficient MEFs were positive for Ki67, a marker expressed specifically in dividing cells, when compared with their corresponding WT counterparts (Figure 1c). Likewise, Ras $^{\mathrm{V} 12} / \mathrm{E} 1 \mathrm{~A}$-transformed Trib3 ${ }^{-/}$MEFs formed four times the number of colonies in soft agar (Figure 1d) as their corresponding WT MEFs. Moreover, this behavior was abolished by re-expressing an HA-tagged form of TRIB3 in Trib3-deficient cells (Figures $1 \mathrm{c}$ and $\mathrm{d}$ ), which strongly supports that the enhanced tumorigenic features of Trib3deficient cells rely solely on the inactivation of this pseudokinase. Furthermore, the onset of tumor xenografts generated by subcutaneous injection of Trib3-deficient Ras ${ }^{\mathrm{V} 12} / \mathrm{E} 1 \mathrm{~A}$ transformed MEFs in the flank of nude mice was remarkably accelerated compared with that of tumors generated with their WT counterparts (Figure 1e).

Of note, TRIB3 is focally deleted in tumors of epithelial origin and in breast cancer patients (tumorscape data set; Supplementary Table SI). In addition, analysis of gene expression profiles of cancer patients from published studies reveals that TRIB3 mRNA levels are downregulated in different tumor types (Supplementary Tables SII and SIII). Therefore we next investigated the effect of the genetic inhibition of TRIB3 in human cancer cell lines. In line with the results obtained with Trib3-deficient MEFs, knockdown of TRIB3 strongly enhanced the ability to form colonies in soft agar of breast carcinoma (BT474) and hepatocellular carcinoma (HepG2) cells lines (Figure 2a). Moreover, TRIB3 silencing increased the number of BT474 and HepG2 cells obtained after several days in culture (Supplementary Figures S2a-b). Furthermore, stable silencing of TRIB3 both accelerated tumor onset and enhanced the rate of tumor growth of xenografts generated with BT474 cells (Figure 2b) and accelerated the onset of HepG2 cell-generated tumors (Figure 2c). Taken together, these observations strongly support that loss of TRIB3 enhances the tumorigenic capacity of different types of cancer cells.

Genetic deletion of Trib3 accelerates the progression of mouse skin papillomas to a more aggressive phenotype. As a first approach to investigate the role of TRIB3 in the control of cancer progression, we analyzed the expression of this pseudokinase in several mouse skin carcinoma cell lines exhibiting different degrees of differentiation and aggressiveness. Specifically, we studied the expression of Trib3 in the MSC11B9/MSC11A5 and CarC-R/CarC cell lines, as they constitute homogenous cellular systems of malignant progression (MSC11B9 and MSC11A5 cells were derived, respectively, from the squamous component and the more undifferentiated region of the same carcinoma; ${ }^{20}$ and CarC-R is a minority population of less aggressive epithelial cells isolated from the fusocellular carcinoma cell line CarC. ${ }^{21}$ ). Trib3 expression was much lower in MSC11A5 and CarC cells than in their corresponding more differentiated counterparts (Figure 3a). Likewise, Trib3 levels were lower in the spindle cell carcinoma cell line CarB when compared with those of PDV cells (derived from a squamous cell carcinoma cell line) (Figure 3a). Moreover, comparison of Trib3 mRNA levels of normal skin, papillomas and skin carcinomas from mice revealed that Trib3 expression correlated inversely with the aggressiveness of the lesion (Figure 3b). These observations support that downregulation of Trib3 expression is associated with a more aggressive phenotype in mouse epithelial skin carcinomas.

Based on these observations, we therefore next investigated the effect of Trib3 genetic inactivation on the progression of the lesions observed in mice subjected to the two-stage skin carcinogenesis model as this is one of the best established paradigms for studying the mechanisms underlying malignant transformation in vivo. ${ }^{22}$ Skin tumors were induced in Trib3 $^{+1+}$ and Trib3 $^{-1-}$ mice of a mixed C57BL6/J 129/Ola background (see Supplementary Methods for details) by topical treatment with a single dose of 7,12-dimethylbenz[a]anthracene (DMBA) followed by a repeated administration of the phorbol ester 12-O-tetradecanoylphorbol-13-acetate (TPA) for 20 weeks. After DMBA/TPA challenge, we observed no differences between Trib3 $^{+/+}$and Trib3 ${ }^{-1-}$ mice either in the latency (time from treatment initiation to tumor development) or the size (data not shown) of the skin lesions. However, the frequency of malignant conversion, as determined by the percentage of papillomas that progressed into a more aggressive phenotype (squamous papillomas or squamous papillomas with dysplasia), was significantly higher in Trib3 ${ }^{-1}$ mice than in their corresponding WT littermates after 20 or 32 weeks of the initiation of the treatment (Figures $3 c$ and $d$ ). Statistically significant differences were not found between Trib3 ${ }^{+/+}$and Trib3 ${ }^{-1}$ mice in the frequency of 'in situ' or squamous cell carcinomas (Supplementary Figure S3).

Loss of TRIB3 enhances the phosphorylation of AKT in cancer cells via mTORC2. TRIB3 has been shown to interact with and inhibit $\mathrm{AKT}$, and dysregulation of this kinase has a crucial role in cancer generation and progression. ${ }^{23}$ Therefore we next investigated whether the enhanced tumorigenic properties of cells in which TRIB3 has been genetically inhibited rely on an enhanced stimulation of the AKT signalling pathway. In line with this idea, RasV ${ }^{12} / E 1 A-$ transformed Trib3-deficient MEFs, as well as breast carcinoma (BT474) and hepatocellular carcinoma (HepG2) cell lines in which TRIB3 had been knocked down, exhibited an enhanced phosphorylation of AKT (Figure $4 a$ and Supplementary Figure S4a). Moreover, the increase triggered by the genetic inhibition of Trib3 in the number of 
a

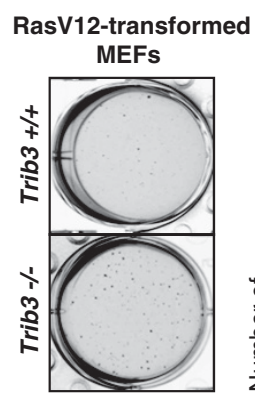

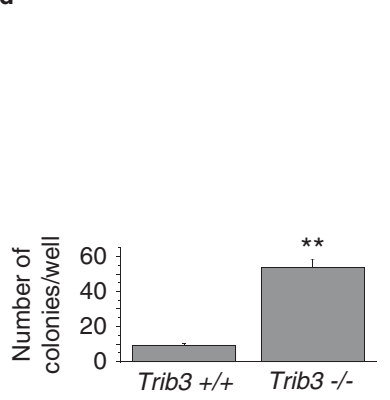

b

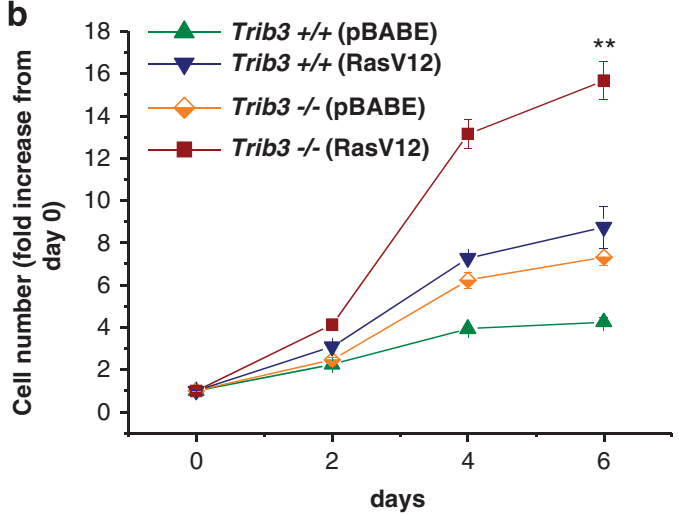

C
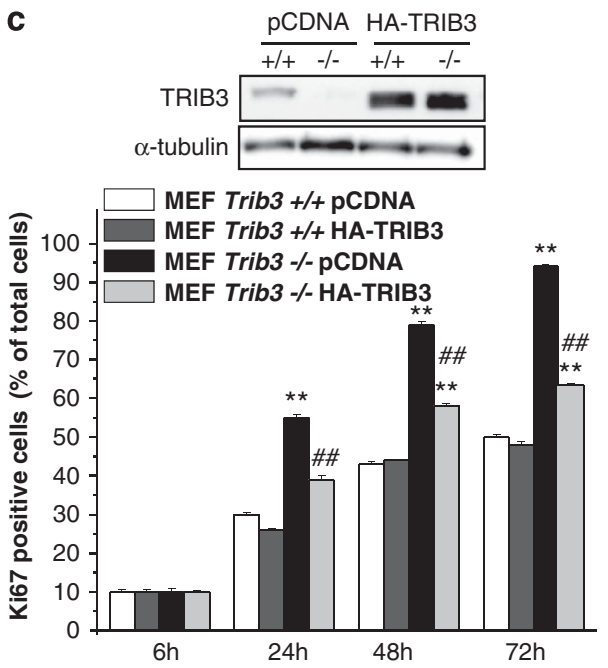

d
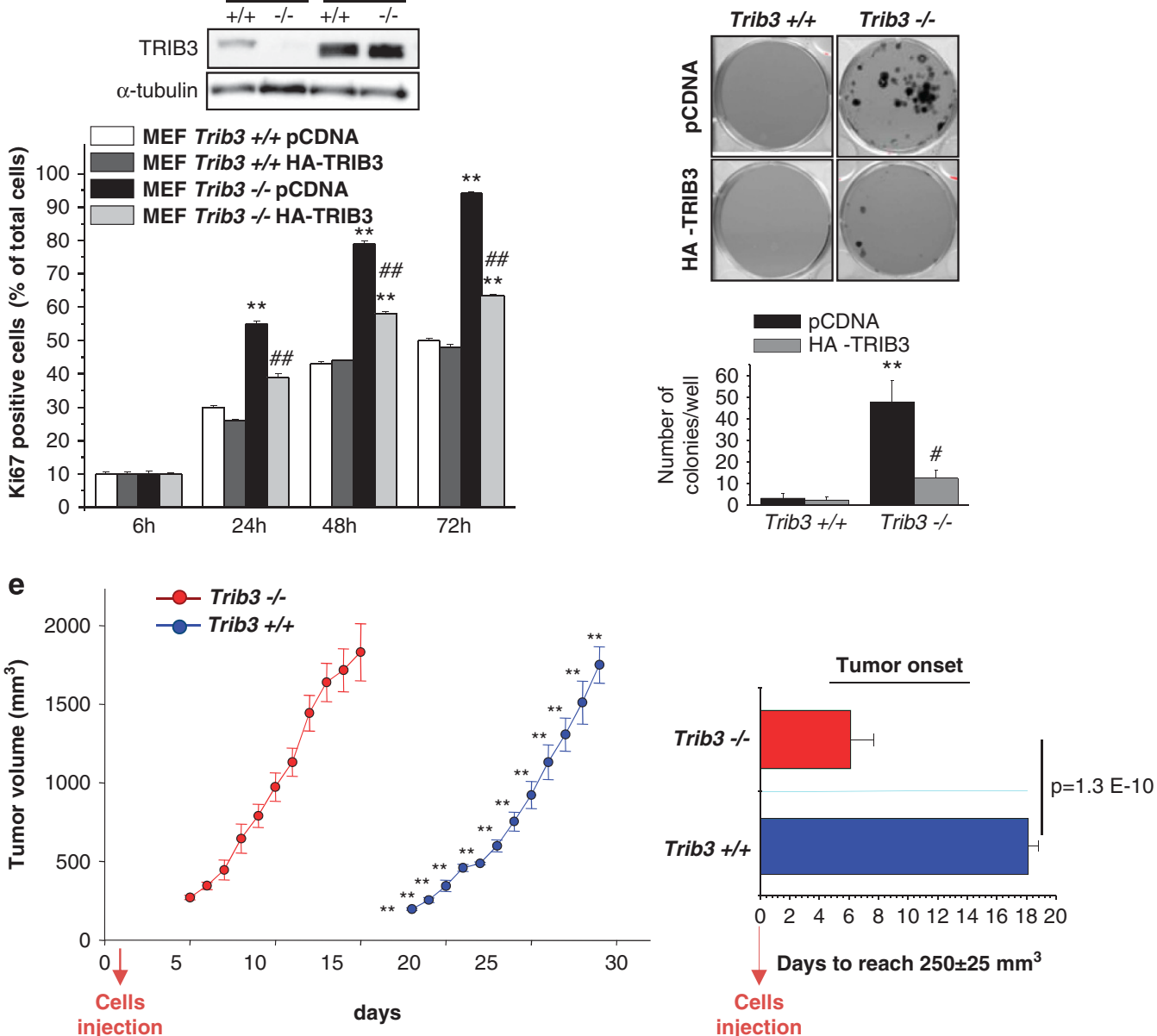

Figure 1 Genetic inhibition of TRIB3 facilitates oncogene transformation and enhances the tumorigenicity of transformed embryonic fibroblasts. (a) Effect of RasV ${ }^{12}$ expression on the ability of Trib3 ${ }^{+1+}$ and Trib3 ${ }^{-1-}$ MEFs to form colonies in soft agar (cells were transduced with a retroviral vector encoding Ras ${ }^{\mathrm{V} 12}$ ) (mean \pm S.D.; $n=3$; ${ }^{* *} P<0.01$ from Trib3 ${ }^{+1+}$ MEFs). (b) Effect of transduction with a retroviral vector encoding Ras ${ }^{12}$ or with the empty retroviral vector pBABE on Trib3 ${ }^{+1+}$ and Trib3 ${ }^{-1-}$ MEF cell number. Data correspond to the number of cells at each time point (as determined by crystal violet staining) relative to the number of cells at day 0 for each experimental condition (mean \pm S.D.; $n=3$; ${ }^{\star *} P<0.01$ from RasV ${ }^{12}$-Trib3 ${ }^{+1+}$ MEFs). (c) Effect of HA-TRIB3 expression on the percentage of Ki67-positive RasV $V^{12} / E 1 A$ transformed Trib3 ${ }^{+1+}$ and Trib3 ${ }^{-1-}$ MEFs. Data correspond to the percentage of Ki67-positive cells relative to the total number of cells in each experimental condition (mean \pm S.D.; $n=4 ;{ }^{* *} P<0.01$ from empty vector-transfected Trib3 ${ }^{+l+}$ cells and ${ }^{\# \#} P<0.01$ relative to empty vector-transfected Trib3 ${ }^{-l-}$ cells). Upper panel: Western blotting analysis of TRIB3 expression. A representative experiment $(n=4)$ is shown. (d) Effect of HA-TRIB3 expression on the ability RasV ${ }^{12} / \mathrm{E}^{\mathrm{A}} \mathrm{A}$-transformed Trib3 ${ }^{+/+}$and Trib3 $^{-l-}$ MEFs to form colonies in soft agar (mean \pm S.D.; $n=6$; ${ }^{* *} P<0.01$ relative to empty vector-transfected Trib3 ${ }^{+l+}$ cells and ${ }^{\#} P<0.05$ relative to empty vector-

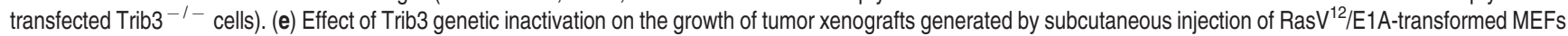
in nude mice. Data correspond to the mean volume change \pm S.E.M. at each time point ( $n=8$ for each condition; $4 \times 10^{6}$ cells were injected in each experimental condition). Right panel: Data correspond to the time elapsed from the injection of the cells until the tumors reach an average volume of $250 \pm 25 \mathrm{~mm}^{3}$ (mean \pm S.E.M.; $n=8$ for each condition; ${ }^{* *} P<0.01$ from tumors generated with Trib3 ${ }^{-1-}$ MEFs) 
a
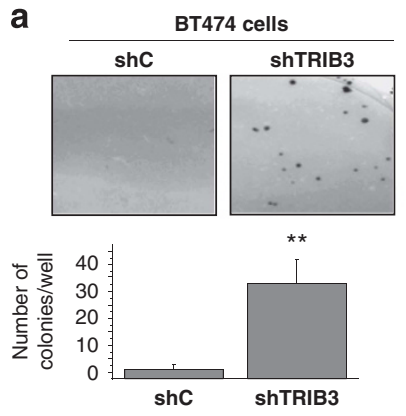

b
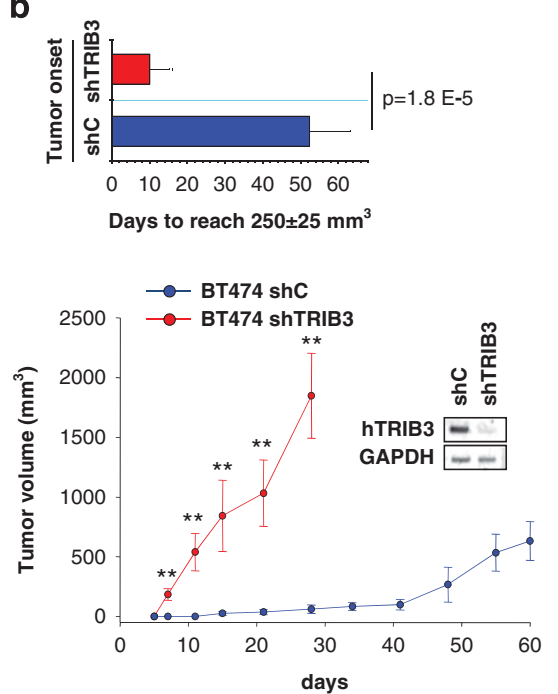
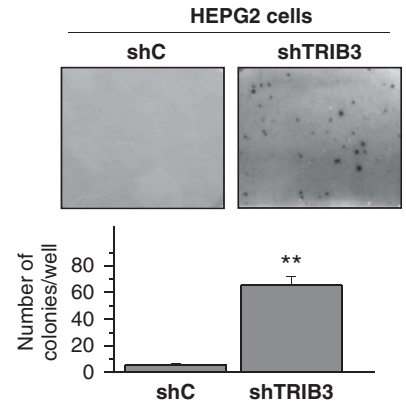

C
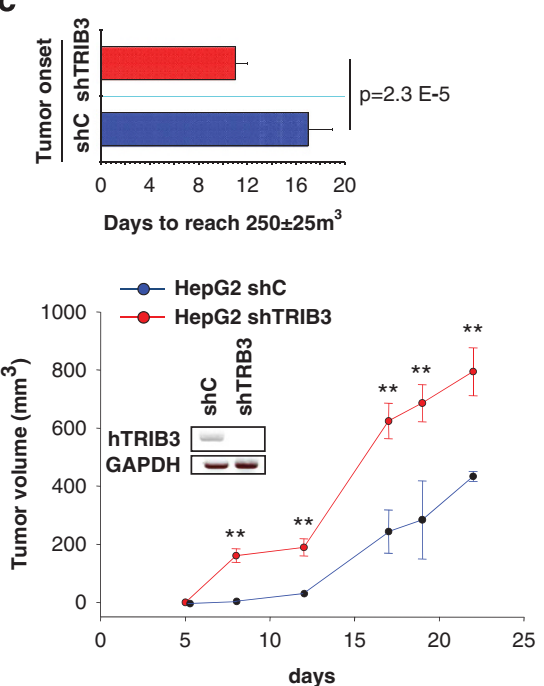

Figure 2 Genetic inhibition of TRIB3 enhances the tumorigenicity of cancer cell lines. (a) Effect of TRIB3 stable knockdown on the ability of BT474 (left panel) and HepG2 (right panel) cells to form colonies in soft agar (mean \pm S.D.; $n=3$; ${ }^{* *} P<0.01$ relative to BT474 or HepG2 cells stably transduced with a lentiviral vector encoding a control (non-targeted) shRNA sequence (shC)). ( $b$ and $\mathbf{c}$ ) Effect of TRIB3 stable knockdown on the growth of tumor xenografts generated by subcutaneous injection of BT474 (b) and HepG2 (c) cells in nude mice. Data on the lower panels correspond to tumor volume (mean \pm S.E.M.; $n=6$ for each condition; ${ }^{*} P<0.05$ and ${ }^{* \star} P<0.01$ from tumors generated with shC BT474 (b) and shC HEPG2 (c) cells). Upper panels: Data correspond to the time elapsed from the injection of the cells until the tumors reach an average volume of $250 \pm 25 \mathrm{~mm}^{3}$ (mean \pm S.E.M.; $n=6$ for each condition). Insets in panels $\mathbf{b}$ and $\mathbf{c}$ show the expression of human TRIB3 mRNA levels (hTRIB3) (as determined by RT-PCR) in tumors generated with shC- and shTRIB3-transduced cells

HepG2 and BT474 cells observed after several days in culture was prevented by the pharmacological inhibition of the Akt pathway (Supplementary Figures $S 4 b-c$ ). Likewise, knockdown of TRIB3 enhanced AKT phosphorylation in BT474- and HepG2- cell-derived tumor xenografts (Figure 4b). Moreover, tumors derived from Ras ${ }^{\mathrm{V} 12} / \mathrm{E} 1 \mathrm{~A}$ transformed Trib3 $^{-1-}$ cells exhibited higher phosphorylation levels of AKT than those derived from their corresponding WT counterparts (Figure 4b). Furthermore, cutaneous squamous papillomas from Trib3 $^{-1-}$ mice also showed higher AKT phosphorylation levels than the papillomas derived from their WT littermates (Figure 4c).

To investigate the mechanism by which the loss of TRIB3 enhances AKT phosphorylation, we analyzed the effect of reexpressing an HA-tagged form of TRIB3 in Trib3-deficient cells. TRIB3 re-expression abolished the enhanced phosphorylation of AKT in Ser 473 but did not modify the phosphorylation of the kinase in Thr 308 (Figure 4d). As AKT is phosphorylated on Ser473 by the mTORC2, ${ }^{24}$ we next investigated whether the enhanced phosphorylation of AKT on Ser 473 observed in Trib3-deficient cells relied on the dysregulation of the phosphorylation of AKT by mTORC2. In line with this idea, silencing of the mTORC2 protein component rapamycin-insensitive companion of mTOR (RICTOR) or incubation with the mTOR kinase inhibitor torin-1 abolished the enhanced phosphorylation of AKT observed in Ras ${ }^{\mathrm{V} 12} / \mathrm{E} 1 \mathrm{~A}$-transformed Trib3 ${ }^{-1-}$ MEFs (Figure $4 \mathrm{e}$ ) as it also did the re-expression of HA-TRIB3 (Supplementary Figure S4d). Moreover, Rictor knockdown prevented the increase (triggered by the genetic inhibition of Trib3) in the number (Figure 4f) and clonogenicity (Figure 4g) of Ras ${ }^{\mathrm{V} 12} / \mathrm{E} 1 \mathrm{~A}$-transformed MEFs, thus suggesting that the tumorigenic properties of Trib3-deficient cells rely on the enhanced phosphorylation of AKT by mTORC2.

Loss of Trib3 enhances the incidence of premalignant and malignant lesions in Pten ${ }^{+/-}$mice. To investigate the relevance of the TRIB3-dependent regulation of AKT in cancer, we next analyzed the in vivo effect of the genetic inactivation of Trib3 together with that of the tumor-suppressor gene phosphatase and tensin homolog (PTEN). PTEN is a highly mutated and frequently lost tumor suppressor in cancer that negatively regulates the activation of the PI3K pathway through the dephosphorylation of phosphatidylinositol $3,4,5$ 


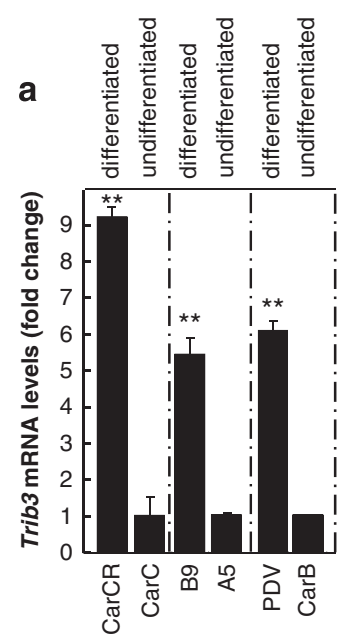

d

Trib3 +/+
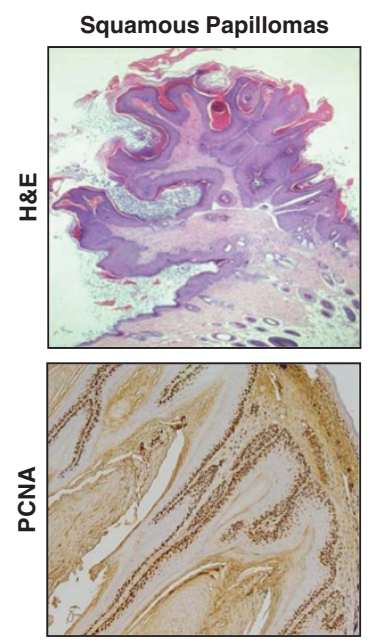

b

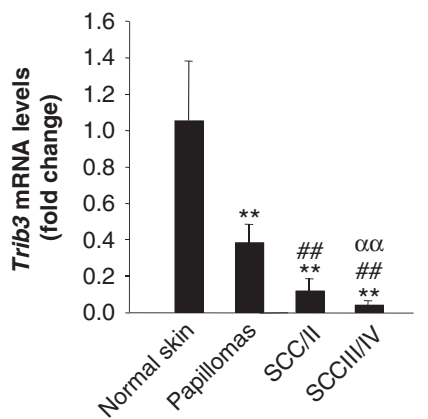

Trib3 -/-

Squamous Papillomas with severe epithelial dysplasia
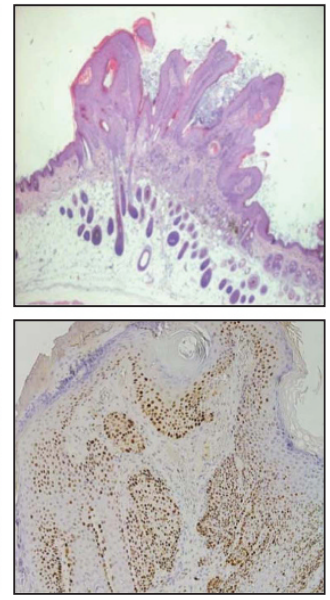

C
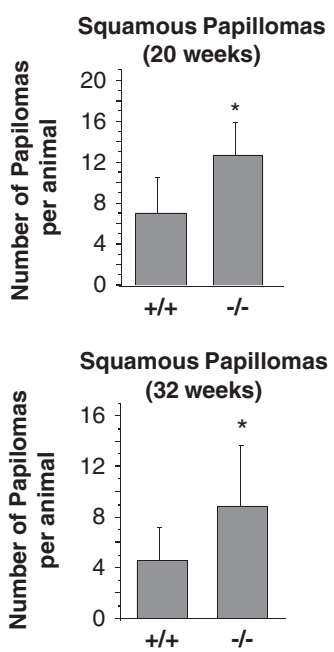

Squamous Papillomas with dysplasia

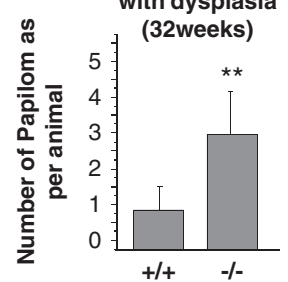

Figure 3 TRIB3 genetic inactivation accelerates the malignant progression of mouse skin papillomas. (a) TRIB3 mRNA levels as determined by real-time quantitative PCR in different mouse skin cancer cell lines. Data are expressed as the mean fold-increase \pm S.D. relative to the corresponding less differentiated cell lines $\left(n=3\right.$; ${ }^{* *} P<0.01$ compared with undifferentiated cells). (b) TRIB3 mRNA levels as determined by real-time quantitative PCR in samples derived from normal skin, papillomas, squamous cell carcinomas (SCC) grades I and II (SCCI/I) and SCC grades III and IV (SCCII//V) obtained from mice subjected to the two-stage model of skin carcinogenesis. Data correspond to TRIB3 mRNA levels and are expressed as the mean fold change \pm S.D. relative to normal skin $(n=8,8,6$ and 6 for normal skin, papillomas, SCCI/II and SCCIII/IV, respectively; ${ }^{\star \star} P<0.01$ from normal skin; ${ }^{\# \# ~} P<0.01$ from papillomas and $\alpha \alpha P<0.01$ from SCC I/II samples). (c) Effect of TRIB3 genetic inactivation on the degree of differentiation and aggressiveness of the skin lesions appeared in Trib3 ${ }^{-1-}$ and Trib3 ${ }^{+l+}$ mice subjected to the two-stage skin carcinogenesis protocol (Animals were killed 20 or 32 weeks after the administration of the DMBA treatment and subsequently subjected to histopathological analyses). Data are expressed as the mean number of squamous papillomas or squamous papillomas with dysplasia per animal \pm S.E.M. (week 20: six Trib3 $3^{+1+}$ and five Trib3 ${ }^{-1-}$ mice; week 32 : seven Trib3 ${ }^{+1+}$ and nine Trib3 ${ }^{-1-}$ mice; ${ }^{* \star} P<0.01$ or ${ }^{*} P<0.05$ from Trib3 ${ }^{+1+}$ animals). (d) Representative images of hematoxilin/eosin (upper panel, $\left.\times 2\right)$ and PCNA staining (lower panel, $\times 10$ ) of squamous papillomas and squamous papillomas with severe dysplasia observed in Trib3 ${ }^{+/+}$and Trib3 ${ }^{-1-}$ mice. See also Supplementary Fig S3

trisphosphate. ${ }^{25}$ Importantly, studies in mice have demonstrated that PTEN is a haplo-insufficient tumor suppressor, as genetic deletion of one copy of the gene is sufficient to unleash oncogenic responses in different organs. $^{26}$ Accordingly, Pten $^{+1-}$ mice exhibit an enhanced activation of the AKT pathway and spontaneously develop malignant lesions in different tissues, including prostate, adrenal glands and thyroid. $^{27}$ Therefore we crossed Trib3 ${ }^{-1-}$ mice with Pten $^{+1-}$ mice to study whether loss of Trib3 results in an acceleration of these lesions. Eight-month-old animals were killed, and histopathological analyses were performed to analyze the presence of premalignant and malignant lesions in different tissues. Trib3 ${ }^{-1}$ mice exhibited a very low frequency of lesions in the different tissues analyzed. However, Pten $^{+/-}$Trib3 $^{+/-}$and Pten ${ }^{+/-}$Trib3 $^{-/-}$mice showed a higher frequency of premalignant and malignant lesions than Pten $^{+/-}$mice in prostate, adrenal glands and thyroid (Table 1, Figure $5 \mathrm{a}$ and Supplementary Figure S5). Specifically, $100 \%$ of Pten $^{+/-}$Trib3 $^{-1-}$ versus $50 \%$ of Pten $^{+/-}$mice developed prostatic intraepithelial neoplasia. Likewise, $67 \%$ of Pten $^{+/-}$ Trib3 $^{-1-}$ versus $37 \%$ of Pten $^{+/}$mice showed hyperplasia of the adrenal glands, and $11 \%$ of Pten ${ }^{+1-}$ Trib3 $^{-1-}$ versus $0 \%$ of Pten ${ }^{+/-}$mice developed pheochromocytomas (Figure 5a). Moreover, AKT phosphorylation was increased 
in hyperplasic adrenal medullas of Trib3 $^{-1-}$ Pten $^{+1-}$ animals when compared with the same lesion in $\mathrm{Pten}^{+1-}$ mice (Figure 5b). These findings strongly support that loss of Trib3 accelerates the formation of premalignant and malignant lesions in Pten $^{+/-}$mice.
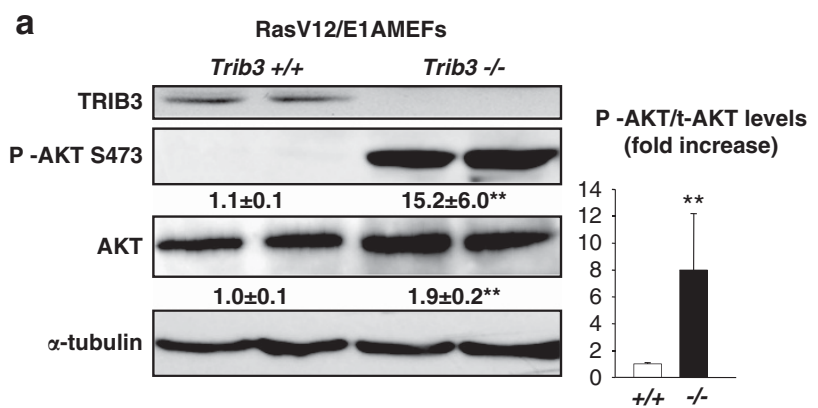

C

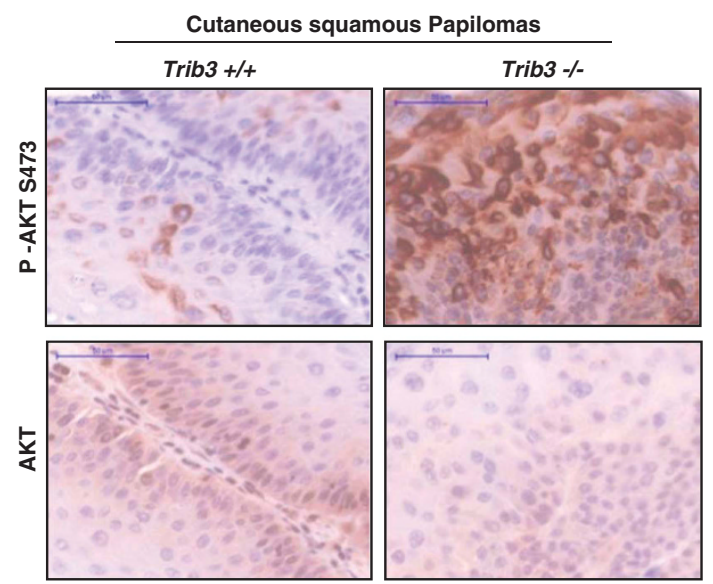

e

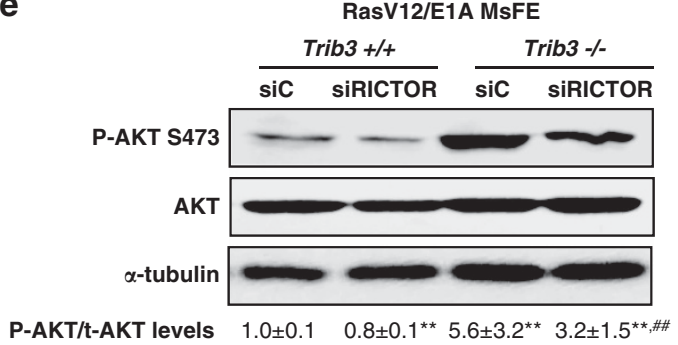

f

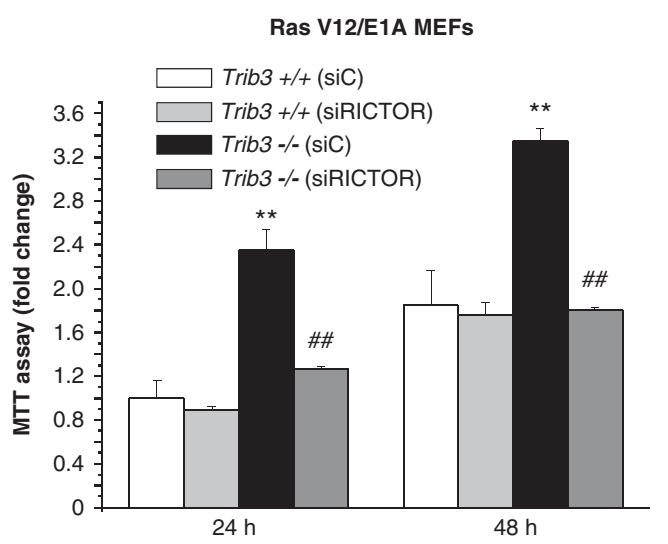

Loss of Trib3 promotes FOXO inactivation. Next, we investigated the targets of AKT that are involved in the enhanced tumorigenic features of cells in which TRIB3 has been genetically inhibited. Remarkably, genetic inhibition of Trib3 led to an enhanced phosphorylation of some of the

b

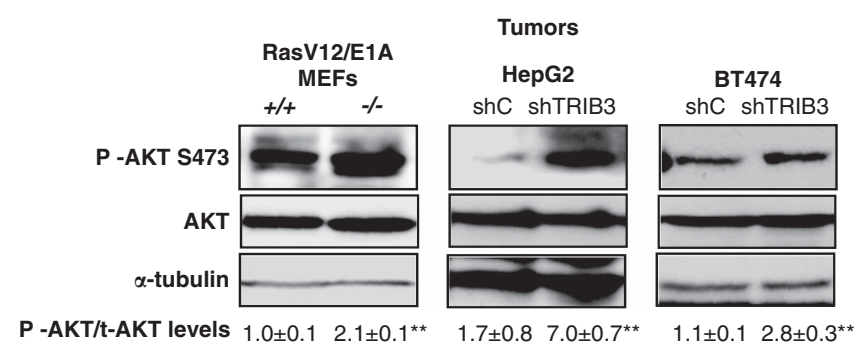

d
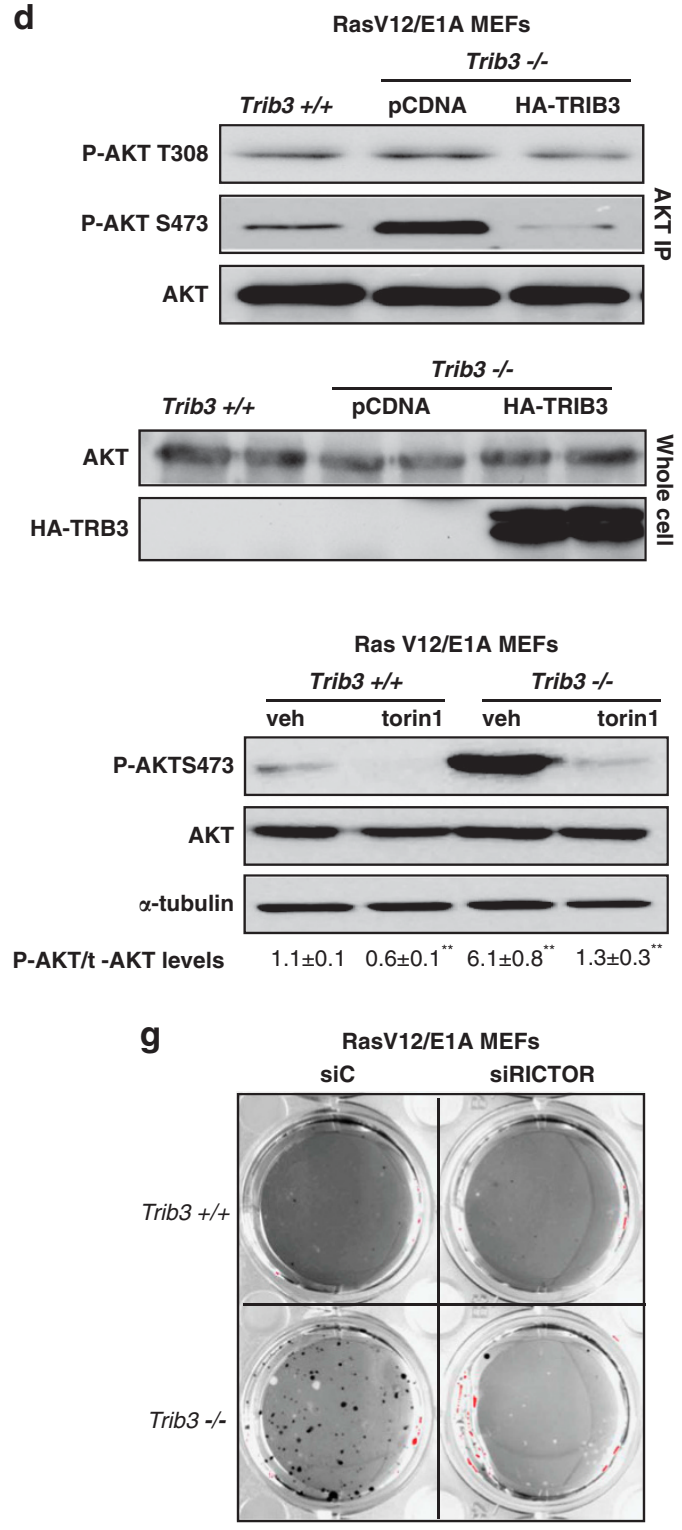
downstream targets of AKT - namely the transcription factor Forkhead box $\mathrm{O} 3$ (FOXO3) and the $\mathrm{BH} 3$-only protein BCL2associated agonist of cell death (BAD) - but not of others such as glycogen synthase kinase 3 (GSK3) or proline-rich AKT substrate (PRAS40) (Figure 6a and Supplementary Figure S6a). Likewise, phosphorylation of FOXO3 (but not of the ribosomal protein S6 - whose phosphorylation correlates under many cellular settings with activation of the mTORC1 complex) was enhanced in TRIB3-silenced BT474 and HepG2 cells (Supplementary Figure S6b).

We therefore next investigated whether the enhanced phosphorylation of FOXO3 and BAD observed in TRIB3-deficient cells

Table 1 Loss of TRIB3 accelerates the formation of premalignant and malignant lesions in Pten $^{+/-}$mice

\begin{tabular}{|c|c|c|c|c|c|c|}
\hline & $\begin{array}{c}\text { Wild type } \\
n=14\end{array}$ & $\begin{array}{c}\text { Trib3 }{ }^{+1-} \\
\mathrm{n}=9\end{array}$ & $\begin{array}{c}\text { Trib3 }-1- \\
\mathrm{n}=12\end{array}$ & $\begin{array}{c}\text { Pten }{ }^{+1-} \\
\mathrm{n}=8\end{array}$ & $\begin{array}{l}\text { Pten }{ }^{+} \text {- } \\
\text { Trib3 }{ }^{+1-} \\
\mathrm{n}=14\end{array}$ & $\begin{array}{c}\text { Pten }{ }^{+1-} \\
\text { Trib3 }-1- \\
\mathrm{n}=9\end{array}$ \\
\hline \multicolumn{7}{|l|}{ Lymph node } \\
\hline Lymphoid hyperplasia submandibular & $0 \%$ & $0 \%$ & $0 \%$ & $62 \%$ & $57 \%$ & $67 \%$ \\
\hline Lymphoid hyperplasia abdominal ganglea & $0 \%$ & $0 \%$ & $0 \%$ & $0 \%$ & $0 \%$ & $22 \%$ \\
\hline \multicolumn{7}{|l|}{ Thyroid } \\
\hline Hyperthyroidism & $0 \%$ & $11 \%$ & $0 \%$ & $12 \%$ & $64 \%$ & $55 \%$ \\
\hline Thyroid adenoma & $0 \%$ & $0 \%$ & $0 \%$ & $0 \%$ & $14 \%$ & $11 \%$ \\
\hline \multicolumn{7}{|l|}{ Liver } \\
\hline Lymphoid infiltrates & $0 \%$ & $0 \%$ & $8 \%$ & $12 \%$ & $0 \%$ & $0 \%$ \\
\hline Steatohepatitis & $0 \%$ & $0 \%$ & $0 \%$ & $0 \%$ & $7 \%$ & $11 \%$ \\
\hline \multicolumn{7}{|l|}{ Intestine } \\
\hline Adenoma & $0 \%$ & $0 \%$ & $0 \%$ & $12 \%$ & $7 \%$ & $11 \%$ \\
\hline \multicolumn{7}{|l|}{ Spleen } \\
\hline \multirow[t]{2}{*}{ Lymphoid hyperplasia } & $0 \%$ & $0 \%$ & $0 \%$ & $0 \%$ & $0 \%$ & $11 \%$ \\
\hline & $\begin{array}{c}\text { Wild type } \\
n=9\end{array}$ & $\begin{array}{c}\text { Trib3 }{ }^{+1-} \\
\mathrm{n}=5\end{array}$ & $\begin{array}{c}\text { Trib3 }^{-/-} \\
\mathrm{n}=4\end{array}$ & $\begin{array}{c}\text { Pten }{ }^{+1-} \\
\mathrm{n}=4\end{array}$ & $\begin{array}{c}\text { Pten }{ }^{+}- \\
\text {Trib3 }{ }^{+1-} \\
\mathrm{n}=12\end{array}$ & $\begin{array}{c}\text { Pten }{ }^{+1-} \\
\text { Trib3 }-1- \\
\mathrm{n}=3\end{array}$ \\
\hline \multicolumn{7}{|l|}{ Anterior prostate } \\
\hline Hyperplasia & $11 \%$ & $0 \%$ & $0 \%$ & $75 \%$ & $100 \%$ & $100 \%$ \\
\hline Adenoma & $0 \%$ & $0 \%$ & $0 \%$ & $25 \%$ & $36 \%$ & $67 \%$ \\
\hline PIN & $0 \%$ & $0 \%$ & $0 \%$ & $50 \%$ & $63 \%$ & $33 \%$ \\
\hline \multicolumn{7}{|l|}{ Dorsal prostate } \\
\hline Hyperplasia & $11 \%$ & $0 \%$ & $0 \%$ & $50 \%$ & $83 \%$ & $100 \%$ \\
\hline Adenoma & $0 \%$ & $0 \%$ & $0 \%$ & $0 \%$ & $25 \%$ & $0 \%$ \\
\hline PIN & $0 \%$ & $0 \%$ & $0 \%$ & $50 \%$ & $75 \%$ & $100 \%$ \\
\hline
\end{tabular}

Incidence of premalignant and malignant lesions (as determined by histopathological analysis) in different tissues and organs of 8 -month-old wild-type, Trib3 $+/-$,

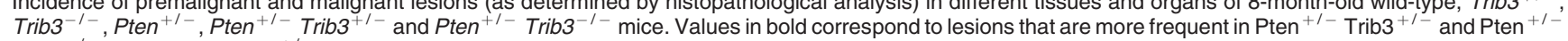
Trib3 $^{-l-}$ mice than in Pten ${ }^{+l-}$ mice.

Figure 4 Loss of TRIB3 enhances the phosphorylation of AKT in cancer cells via mTORC2. (a) Effect of TRIB3 genetic inactivation on AKT phosphorylation of RasV ${ }^{12}$, E1A-transformed MEFs. Values below each of the western blottings correspond to the densitometric analysis of the ratio Phospho-Akt (P-Akt)/tubulin and total Akt (t-Akt)/ tubulin, respectively, and are expressed as the mean fold change \pm S.D. relative to Trib3 ${ }^{+/+}$cells. Data on the right panel correspond to the P-Akt/t-Akt ratio and are expressed as the mean fold change \pm S.D. relative to Trib3 ${ }^{+/+}$cells. $\left(n=4 ;{ }^{* *} P<0.01\right.$ from Trib3 ${ }^{+/+}$cells) (b) Effect of TRIB3 genetic inactivation (left panel) or stable knockdown (middle and right panels) on AKT phosphorylation as determined by western blotting analysis of tumor xenografts generated by subcutaneous injection of Ras $V^{12}$ / E1A-transformed MEFs (left panel), HepG2 (middle panel) and BT474 (right panel) cells in nude mice. Data correspond to the densitometric analysis of AKT phosphorylation expressed as the P-Akt/t-Akt ratio relative to Trib3 ${ }^{+/+}$-MEFs (left panel), shC BT474 (middle panel) or shC BT474 (right panel) tumors $\left(n=3\right.$; mean \pm S.D.) ${ }^{* *} P<0.01$ from tumors generated with Trib3 ${ }^{+/+}$MEFs, shC HEPG2 or shC BT474 cells). (c) Effect of TRIB3 genetic inactivation on AKT phosphorylation of cutaneous squamous papillomas. Representative images $(\times 40)$ of P-AKT and t-AKT immunohystochemistry staining from three cutaneous squamous papillomas per experimental condition are shown. (d) Effect of HA-TRIB3 expression on the phosphorylation of Akt on Thr 308 and Ser 473 of RasV ${ }^{12} / E 1 A$-transformed Trib3 ${ }^{-1-}$ MEFs ( $n=3$, a representative experiment is shown). Upper panel: AKT was immunoprecipitated from cell extracts obtained from RasV ${ }^{12} / E 1 A$-transformed Trib3 $3^{+1+}$ or Trib3 $3^{-1-}$ MEFs transfected with an empty vector (pCDNA) or a plasmid encoding HA-TRIB3. Lower panel: Analysis of total AKT and HA-TRIB3 levels in the corresponding whole cell extracts (samples were loaded by duplicate). (e) Effect of RICTOR knockdown (left panel) or incubation in the presence of torin1 ( $250 \mathrm{nM}, 18 \mathrm{~h}$; right panel) on the phosphorylation of AKT on Ser 473 of RasV ${ }^{12} /$ E1A-transformed Trib3 $3^{+1+}$ or Trib3 ${ }^{-1-}$ MEFs ( $n=3$; a representative western blotting analysis is shown). Values below each of the western blottings correspond to the P-Akt/t-Akt ratio and are expressed as the mean fold change \pm S.D. relative to shC, Trib3 ${ }^{+/+}$(left panel) or veh-treated Trib3 ${ }^{+/+}$(right panel) cells. $\left(n=3\right.$; ${ }^{* *} P<0.01$ from shC Trib3 ${ }^{+/+}$(left panel) or veh-treated shC Trib3 ${ }^{+/+}$(right panel) cells and ${ }^{\# \#} P<0.01$ from shC Trib3 ${ }^{-1-}$ cells (left panel) or from veh-treated Trib3 ${ }^{-1-}$ (right panel) cells) (f) Effect of RICTOR knockdown on the number (as estimated by the MTT test) of RasV ${ }^{12} / E 1$ A-transformed Trib3 ${ }^{+/+}$and Trib3 ${ }^{-1-}$ MEFs. Data are expressed as themean fold increase \pm S.D. relative to $\mathrm{siC}$ Trib3 ${ }^{+1+}$ MEFs at $24 \mathrm{~h} ;\left(n=4\right.$; ${ }^{* *} P<0.01$ from siC-transfected Trib3 ${ }^{+1+}$ MEFs; ${ }^{\# \#} P<0.01$ from siC-transfected Trib3 ${ }^{-1-}$ MEFs). (g) Effect of RICTOR knockdown on the ability of RasV ${ }^{12} / E 1 A$-transformed Trib3 ${ }^{+/+}$and Trib3 ${ }^{-1-}$ MEFs to form colonies in soft agar ( $n=3$. Images of one representative experiment are shown). (e-g) Transfection with RICTOR siRNA reduced RICTOR mRNA levels (as determined by quantitative real-time PCR) of Trib3 ${ }^{+/+}$and Trib3 $^{-/-}$cells by $83 \pm 1$ and $91 \pm 2 \%$, respectively $(P<0.01$ from the corresponding siC-transfected RasV12/E1A-MEFs; $n=4)$ 
a

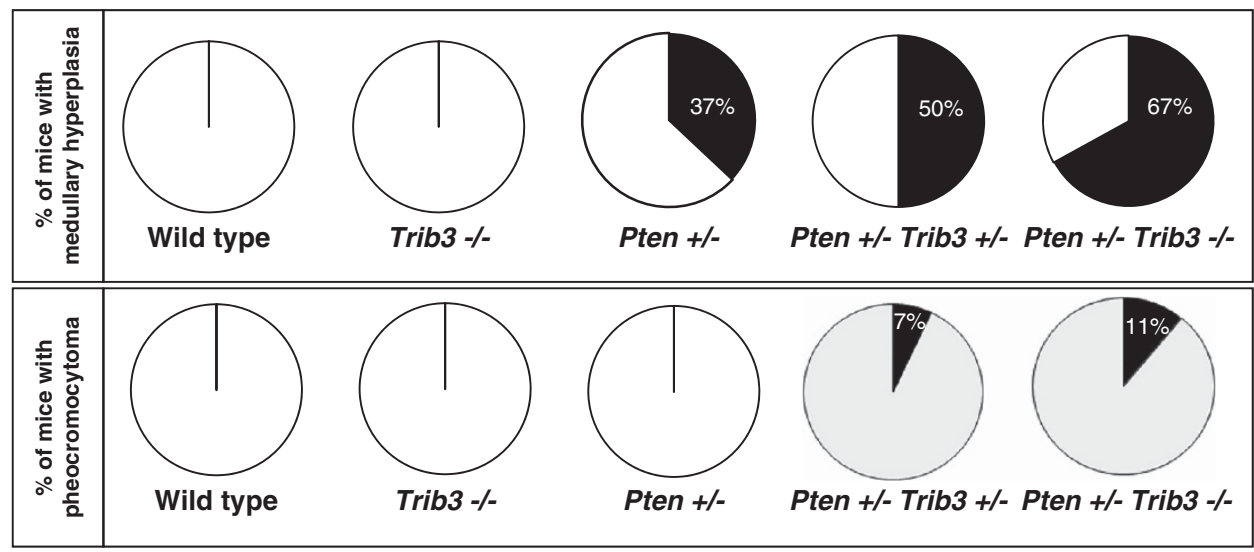

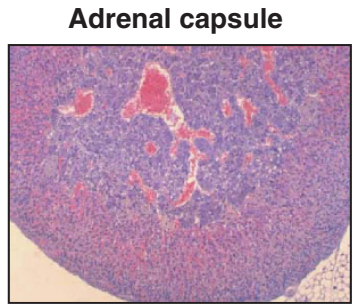

Wild type

b
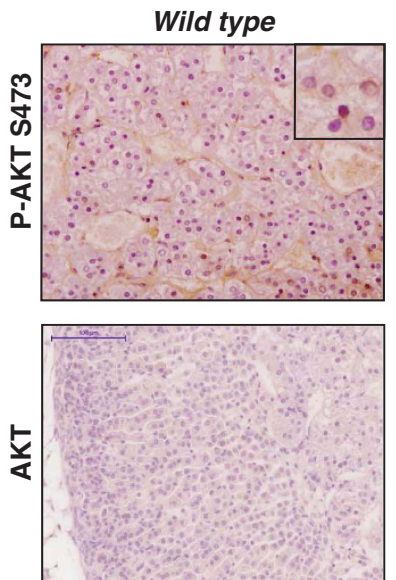

Medullary hyperplasia

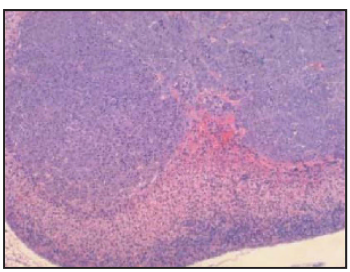

Pten+/-

Medullary hyperplasia

Pten $+/-$
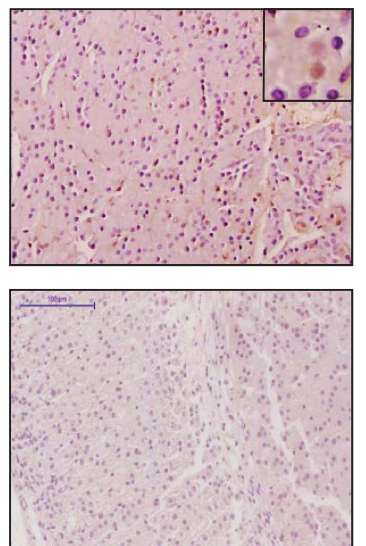

Pheocromocytoma

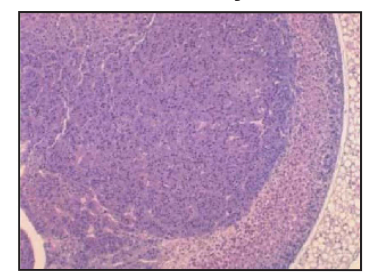

Pten+/- Trib3-/-

Pten +/- Trib3 -/-
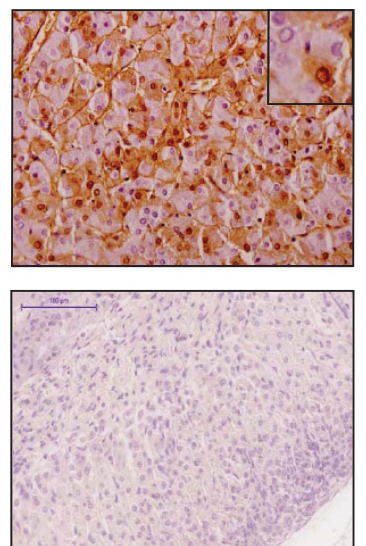

Figure 5 Loss of TRIB3 enhances the incidence of premalignant and malignant lesions in Pten ${ }^{+/}$mice. (a) Effect of the genetic inactivation of TRIB3 on the incidence of premalignant and malignant lesions in the adrenal glands of $P \operatorname{ten}{ }^{+1}$ mice. Data are expressed as the percentage of animals of each genotype that exhibit hyperplasia of the adrenal glands (upper panel) or pheocromocytomas (middle panels). Bottom panel: Representative microphotographs $(\times 10)$ of the different lesions observed in the adrenal glands of Pten ${ }^{+1}$ Trib3 ${ }^{+1-}$ and Pten ${ }^{+1}$ Trib3 ${ }^{-1-}$ animals stained with hematoxilin/eosin. (b) Representative microphotographs of anti-phospho AKT and total Akt immunostaining of the adrenal glands of WT, Pten ${ }^{+1-}$ and Pten ${ }^{+1-}$ Trib3 $^{-1-}$ animals. Note that the hyperplasic region located in the medulla of the adrenal gland exhibits strong phospho-AKT immunostaining in Pten ${ }^{+1-}$ Trib3 $^{-l-}$ mice but not in Pten ${ }^{+1-}$ mice $(n=3 ; \times 40)$. Insets show details of the different panels at higher magnification. See also Supplementary Figure S5

relied on the dysregulation of the phosphorylation of AKT by mTORC2. In line with this idea, silencing of the mTORC2 protein component Rictor or incubation with the mTOR kinase inhibitor torin-1 abolished the enhanced phosphorylation of FOXO and BAD observed in Trib3-deficient MEFs at the same extent as the re-expression of HA-TRIB3. By contrast, phosphorylation of Pras40 was not modified in any of those experimental conditions (Figure 6b and Supplementary Figures S6c and d).

It has been widely reported that the FOXO family of transcription factors has an important tumor-suppressing role. $^{28}$ As genetic inhibition of Trib3 enhances FOXO3 phosphorylation - an event that typically leads to the inactivation and the subsequent sequestration in the cytosol of this transcription factor ${ }^{23,29,30}$ - we asked whether the activity of FOXO is altered in Trib3 ${ }^{-1-}$ cells. In line with this idea, FOXO3 exhibited a primarily cytosolic localization in Ras $^{\text {V12/E1A-transformed Trib3 }}{ }^{-1}$ MEFs subjected to serum withdrawal - an insult that typically leads to FOXO3 translocation to the nucleus ${ }^{30}$ (Figure 6c). In contrast, the distribution of $\mathrm{FOXO} 3$ was mainly nuclear in 
a

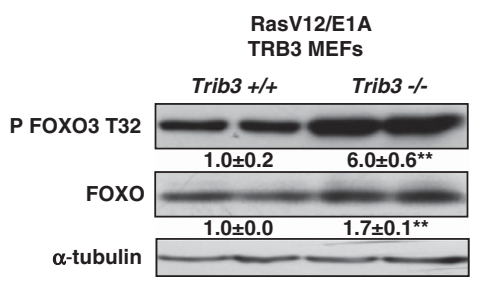

b

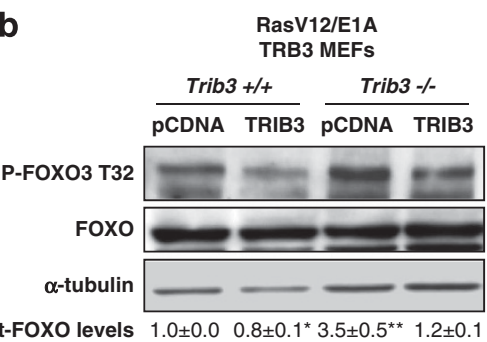

p-FOXO/t-FOXO levels $1.0 \pm 0.0 \quad 0.8 \pm 0.1^{*} 3.5 \pm 0.5^{\star \star} \quad 1.2 \pm 0.1$
C

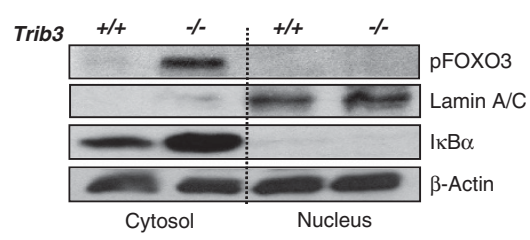

p-FOXO/t-FOXO levels (fold increase)

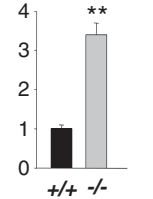

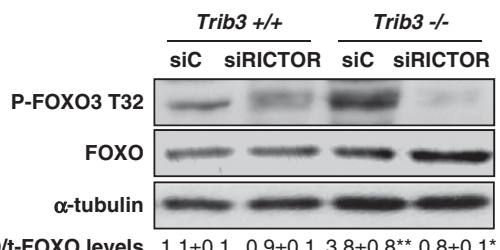

p-FOXO/t-FOXO levels $1.1 \pm 0.1 \quad 0.9 \pm 0.13 .8 \pm 0.8^{* \star} 0.8 \pm 0.1^{\text {* }}$

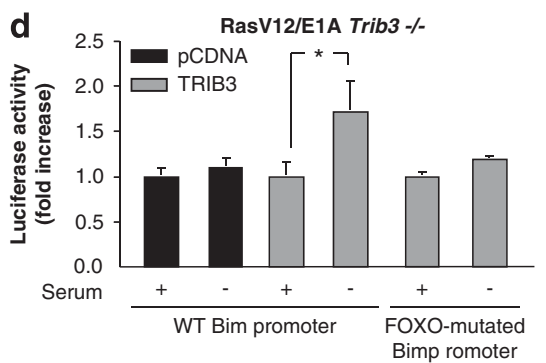

e

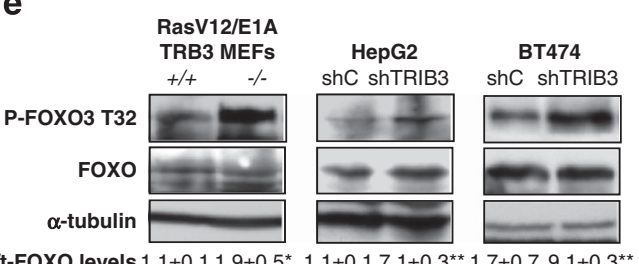

p-FOXO/t-FOXO levels $1.1 \pm 0.11 .9 \pm 0.5^{\star} 1.1 \pm 0.17 .1 \pm 0.3^{\star *} 1.7 \pm 0.79 .1 \pm 0.3^{\star *}$

f

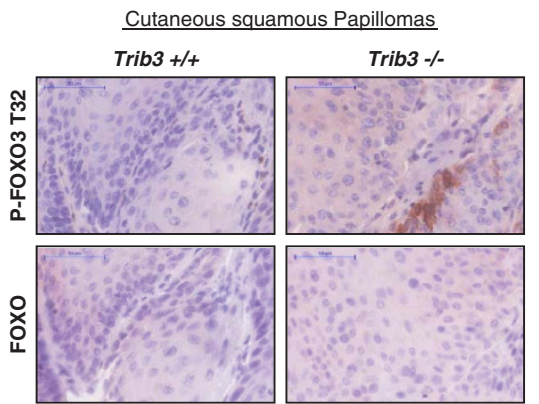

Figure 6 Loss of TRIB3 promotes FOXO inactivation. (a) Effect of TRIB3 genetic inactivation on the phosphorylation of FOXO, of Ras ${ }^{\mathrm{V} 12} / \mathrm{E} 1 \mathrm{~A}$-transformed MEFs. Values below the western blottings correspond to the densitometric analysis of the ratio Phospho-FOXO (P-FOXO)/tubulin and total FOXO (t-FOXO)/tubulin respectively and are expressed as the mean fold change \pm S.D. relative to Trib3 ${ }^{+/+}$cells. Data on the right panel correspond to the P-FOXO/t-FOXO ratio and are expressed as the mean fold change \pm S.D. relative to Trib3 ${ }^{+/+}$cells. $\left(n=4 ;{ }^{* \star} P<0.01\right.$ from Trib3 ${ }^{+/+}$cells) (b) Effect of TRIB3 re-expression (upper panel), and RICTOR knockdown (lower panel) on the phosphorylation of FOXO, of Ras $V^{12} / E 1 A$-transformed Trib3 $3^{+/+}$and Trib3 ${ }^{-/-}$MEFs. Values at the bottom of the panels correspond to the P-FOXO/t-FOXO ratio and are expressed as the mean fold change \pm S.D. relative to $p C D N A$ Trib3 $3^{+/+}$(upper panel) or siC-transfected Trib3 ${ }^{+/+}$(lower panel) MEFs. $\left(n=3 ;{ }^{* *} P<0.01\right.$ and ${ }^{*} P<0.05$ from pCDNA Trib3 ${ }^{+/+}$(upper panel) or siC-transfected Trib3 ${ }^{+/+}$(lower panel) cells. Transfection with RICTOR siRNA reduced RICTOR mRNA levels (as determined by quantitative real-time PCR) of Trib3 ${ }^{+1+}$ and Trib3 $3^{-1-}$ cells by $83 \pm 1$ and $91 \pm 2 \%$, respectively $(n=4 ; P<0.01$ from the corresponding siC-transfected RasV12/E1A$\mathrm{MEFs}$ ). (c) Effect of serum withdrawal on the sub-cellular distribution (as determined by cellular fractionation and subsequent western blotting analysis of cytosolic and nuclear

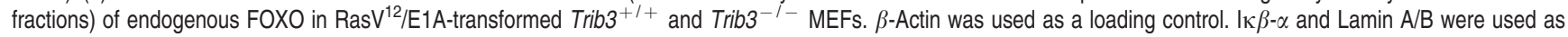
markers of the cytosolic and the nuclear fractions, respectively $(n=3$; a representative experiment is shown). (d) Effect of serum withdrawal on the activity of the Bim promoter of RasV ${ }^{12} /$ E1A-transformed Trib3 ${ }^{-1-}$ MEFs transfected with a vector encoding HA-TRIB3 or a control plasmid and co-transfected with a luciferase reporter vector containing the sequence of the Bim promoter (WT or mutated on the FOXO-binding sites). Data correspond to the luciferase activity and are expressed as the mean fold increase \pm S.D. relative to HA-TRIB3-transfected Trib3 ${ }^{-1-}$ cells incubated in the presence of serum $\left(n=3 ;{ }^{*} P<0.05\right.$ from serum-treated HA-TRIB3-transfected Trib3 ${ }^{-1-}$ cells). (e) Effect of TRIB3 genetic inactivation (e, left panel) or stable knockdown (e, middle and right panels ) on FOXO phosphorylation as determined by western blotting analysis of tumor xenografts generated by subcutaneous injection of RasV ${ }^{12} / E 1 A$-transformed MEFs (left panel), HepG2 (middle panel) and BT474 cells (right panel) in nude mice. Values in the bottom of the panels correspond to the P-FOXO/t-FOXO ratio and are expressed as the mean fold change \pm S.D. relative to Trib3 ${ }^{+/+}$tumors (left panel) or shC HepG2 tumors (middle panel) or shC BT474 tumors (right panel) $\left(n=3\right.$; ${ }^{* *} P<0.01$ or ${ }^{*} P<0.05$ from tumors generated with Trib3 ${ }^{+/+}$MEFs, shC HEPG2 or shC BT474 cells). (f) Effect of TRIB3 genetic inactivation on FOXO3 phosphorylation of cutaneous squamous papillomas. Representative images $(\times 40)$ of phospho-FOXO1/3 or total FOXO3 immunohistochemistry staining from three cutaneous squamous papillomas per experimental condition are shown. See also Supplementary Figure S6

Ras $^{\mathrm{V} 12} /$ E1A-transformed Trib3 ${ }^{+/+}$MEFs upon the same experimental conditions (Figure 6c). Likewise, serum withdrawal - which triggers the upregulation of the Bcl-2 family gene $\mathrm{Bcl}-2$ interacting mediator of cell death (BCL2-like 11 (BIM)) in a FOXO-dependent manner - led to the activation of the Bim promoter in Trib3 $3^{+/+}$but not in Trib3 $3^{-/-}$cells 
(Supplementary Figure S6e). Furthermore, TRIB3 re-expression restored the ability of serum deprivation to activate the Bim promoter in Trib3 ${ }^{-1-}$ cells, and this effect was abolished when the FOXO-binding sites were mutated in this promoter, thus supporting that TRIB3 participates in the regulation of FOXO activity (Figure 6d). To investigate the in vivo relevance of our findings, we next analyzed whether genetic inhibition of TRIB3 affects the phosphorylation of FOXO in tumor samples. Knockdown of TRIB3 enhanced FOXO3 phosphorylation in HepG2- andBT474-cell-derived tumor xenografts (Figure 6e) which occurred in concert with an enhanced expression of a the cell proliferation marker $\mathrm{Ki} 67$ in those tumors (Supplementary Figures S6f and g). Likewise, tumors derived from Ras ${ }^{\mathrm{V} 12} / \mathrm{E} 1 \mathrm{~A}$-transformed Trib3 $^{-1-}$ cells exhibited a higher phosphorylation of FOXO (Figure 6e) that was associated with an enhanced Ki67 immunostaining (Supplementary Figure S6h) compared with tumors derived from their corresponding WT counterparts. Furthermore, cutaneous squamous papillomas from Trib3 $^{-1-}$ mice also showed higher FOXO1/3 phosphorylation levels than the papillomas derived from their WT littermates (Figure 6f).

Loss of Trib3 enhances tumor growth via FOXO inactivation. To study whether the enhanced tumorigenic properties of Trib $^{-1-}$ cells relies on FOXO inhibition, we made use of a construct encoding a form of FOXO3 in which the residues phosphorylated by AKT are mutated to Ala

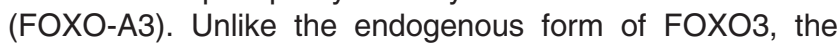
FOXO-A3 mutant is located in the nucleus of Trib3 $^{-1-}$ cells (Figure 7a). Moreover, the expression of FOXO-A3 in Trib3 $^{-1-}$ cells re-established the pattern of FOXO-regulated genes (Supplementary Figure S7a), as well as the ability of serum deprivation to activate the Bim promoter (Supplementary Figure S7b). In addition, FOXO-A3 expression abolished the increase in the number of cells and the enhanced clonogenicity characteristic of $\mathrm{Trib3}^{-1-}$ cells (Figures $7 b$ and $\mathrm{c}$ ).

Therefore, we next analyzed the effect of the stable expression of the FOXO-A3 mutant on the ability of Ras ${ }^{\mathrm{V} 12}$ / E1A-transformed Trib3 ${ }^{-1-}$ MEFs to generate tumor xenografts. As shown in Figure 7d, the growth rate of tumors generated with Foxo-A3-Trib3 ${ }^{-l}$ cells was strongly reduced in comparison with that of Trib3 ${ }^{-1-}$ cells. Likewise, tumors derived from FOXO-A3-Trib3 ${ }^{-1}$ cells exhibited a decreased expression of Ki67 when compared with those generated with Trib3 $^{-1-}$ cells (Figure 7e). Taken together, these observations strongly support that the enhanced tumorigenic properties of Trib3-deficient cells rely - at least in major part - on the enhanced phosphorylation and subsequent inhibition of FOXO transcription factors.

\section{Discussion}

Several observations presented in this work show that TRIB3 has a remarkable tumor-suppressive role. For example, (i) oncogenic transformation is facilitated in MEFs derived from Trib3-deficient mice; (ii) genetic inhibition of TRIB3 enhances proliferation, clonogenicity and the ability to generate tumor xenografts of oncogene-transformed MEFs and of several human cancer cell lines; (iii) loss of TRIB3 enhances the frequency of malignant conversion of papillomas generated in mice subjected to DMBA/TPA treatment; and (iv) loss of TRIB3 enhances the incidence of premalignant and malignant lesions in Pten ${ }^{+/-}$mice.

Our findings demonstrate that the tumor inhibitory role of TRIB3 relies to a large extent on the ability of this pseudokinase to regulate the activity of the AKT pathway. In line with this idea, we found that the genetic inactivation of Trib3 enhances the effect of the deletion of one of the two copies of Pten on the formation of premalignant and malignant lesions in mice. Of note, AKT phosphorylation is increased in hyperplasic adrenal medullas of Pten ${ }^{+/}$Trib3 $^{-1-}$ animals when compared with the same lesion in Pten ${ }^{+/}$mice, thus suggesting that loss of Trib3 produces an increase in the phosphorylation of AKT that contributes to the malignant progression in this tissue. In the same line of reasoning, Trib3 $^{-1-}$ mice subjected to the two-step skin carcinogenesis protocol generated papillomas of a more aggressive phenotype and with higher levels of AKT phosphorylation than papillomas generated in WT littermates. Likewise, tumor xenografts generated with Ras ${ }^{\mathrm{V} 12} / \mathrm{E} 1 \mathrm{~A}$-transformed Trib3 ${ }^{-1-}$ MEFs or with different cancer cell lines in which TRIB3 had been knocked down exhibited an enhanced phosphorylation of AKT when compared with their corresponding WT or control

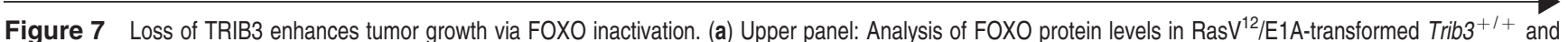
Trib3 $^{-1-}$ MEFs stably transfected with a constitutively active version of FOXO (FOXOA3) or the corresponding empty vector (pBABE). Lower panel: Sub-cellular distribution (as determined by cellular fractionation and subsequent western blotting analysis of cytosolic and nuclear fractions) of FOXO protein in Ras $\mathrm{F}^{12} / \mathrm{E}^{\mathrm{A}} \mathrm{A}$-transformed Trib3 ${ }^{-1}-$ MEFs stably transfected with FOXOA3. $\beta$-Actin was used as a loading control. $1 \kappa \beta-\alpha$ and Lamin $A / B$ were used as markers of the cytosolic and the nuclear fractions, respectively ( $n=3$; a representative experiment is shown). (b) Effect of TRIB3 genetic inactivation and stable expression of FOXOA3 on the number of Ras ${ }^{12} / E 1 A$ transformed MEFs (as estimated by the MTT test) at the indicated time points. Data are expressed as the mean fold change \pm S.D. relative to pBABE-Trib3 $+1+$ cells at $24 \mathrm{~h}$ (mean \pm S.D.; $n=3 ;{ }^{* *} P<0.01$ from pBABE-Trib3 ${ }^{+1+}$ cells; ${ }^{\# \#} P<0.01$ from pBABE-Trib3 ${ }^{-1-}$ cells). (c) Effect of TRIB3 genetic inactivation and stable expression of FOXOA3 on the ability of RasV ${ }^{12} / E 1 A$-transformed MEFs to form colonies in soft agar ( $n=3$. A representative experiment is shown). (d) Effect of FOXOA3 stable expression and TRIB3 genetic inactivation on the growth of tumor xenografts generated by subcutaneous injection of RasV ${ }^{12} / E 1 A$-transformed MEFs in nude mice. Data are expressed as

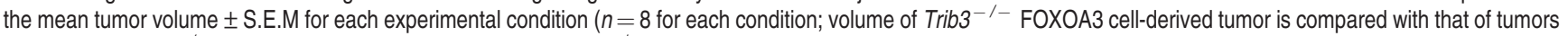
derived from Trib3 ${ }^{-1-}$ pBabe cells; ${ }^{* \star} P<0.01$ from $\mathrm{PBABE}-$ Trib3 ${ }^{-1}-$ cell-derived tumors). Lower panel: Analysis of FOXO protein levels in tumors derived from FOXOA3Trib3 $^{-1-}$ and pBABE-Trib3 ${ }^{-1-}$ cells. (e) Analysis of Ki67 immunostaining in samples from tumors derived from pBABE-Trib3 ${ }^{+I+}$, pBABE-Trib3 ${ }^{-I-}$ and FOXOA3Trib3 $^{-1-}$ cells. Values in the lower right corner of microphotographs correspond to the Ki67-stained area relative to the total number of nuclei in each section and are expressed as the mean fold change relative to pBABE-Trib3 ${ }^{+l+}$ tumors \pm S.D. ${ }^{\star \star *} P<0.01$ from pBABE-Trib3 ${ }^{+I+}$ tumors; $^{\# \#} P<0.01$ from pBABE-Trib3 ${ }^{-I-}$ tumors; 18 sections for each of the 3 dissected tumors for each condition were counted). Representative microphotographs are shown. (f) Proposed model of the mechanism by which TRIB3 controls tumorigenesis. TRIB3 interacts with AKT, which regulates the phosphorylation of the kinase by the mTORC2 complex (left panel). Genetic inhibition of TRIB3 in combination with different oncogenic signals facilitates the hyperphosphorylation of AKT on Ser 473 by the mTORC2 complex and the subsequent hyperphosphorylation and inactivation of the transcription factor FOXO3 (as well as of the BH3-only protein BAD) but not of the other AKT downstream targets. The hyperphosphorylation and inactivation of FOXO is responsible - at least in part - for the enhanced tumorigenic features of TRIB3-deficient cells. See also Supplementary Figure S7 and Supplementary Table SII 
siRNA-transfected counterparts. Taken together, these observations strongly suggest that TRIB3 limits the capacity of AKT to become overactivated in response to oncogenic signals.

Our findings also define the mechanism of the tumorsuppressive activity of TRIB3 downstream of AKT, which relies on the selective regulation of $\mathrm{FOXO} 3$ activity. Thus, reexpression of a mutant form of $\mathrm{FOXO} 3$ in which the residues phosphorylated by AKT have been mutated to Ala abolished the enhanced proliferation and clonogenicity of TRIB3deficient cells and re-established the pattern of FOXOregulated genes characteristic of WT cells. Furthermore, re-expression of this construct decreased proliferation and growth rate of tumors generated with Trib3-deficient cells. Although care should be taken in the interpretation of data derived from the overexpression of a transcription factor,

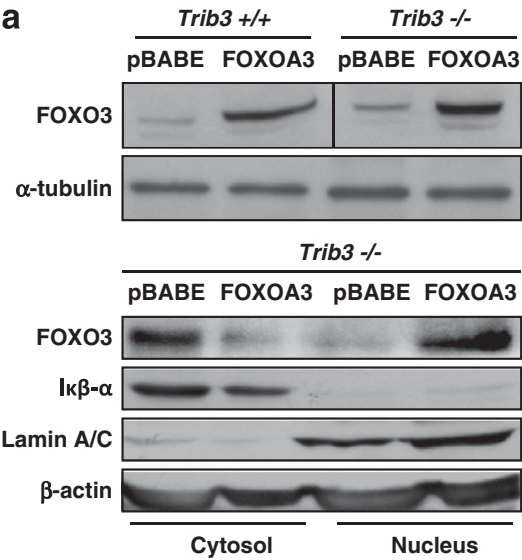

d

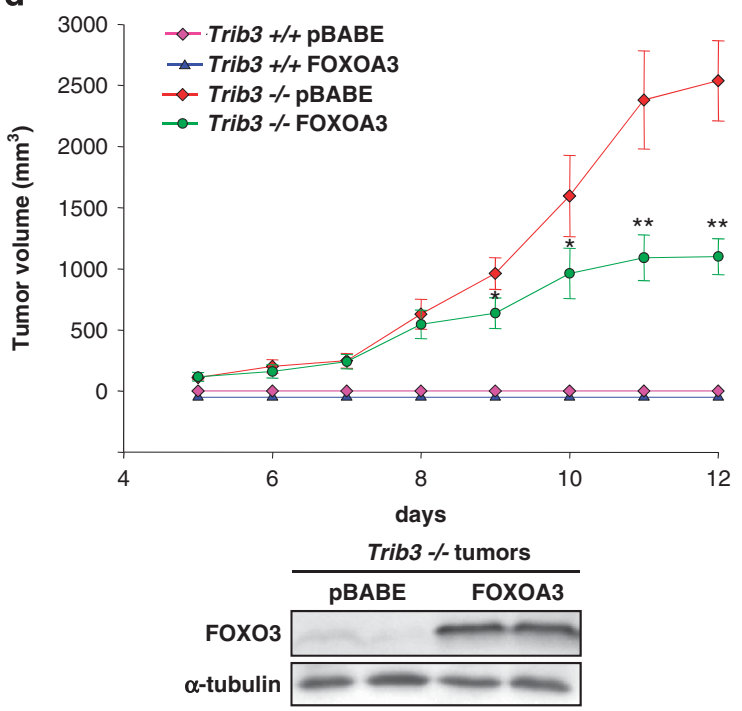

b

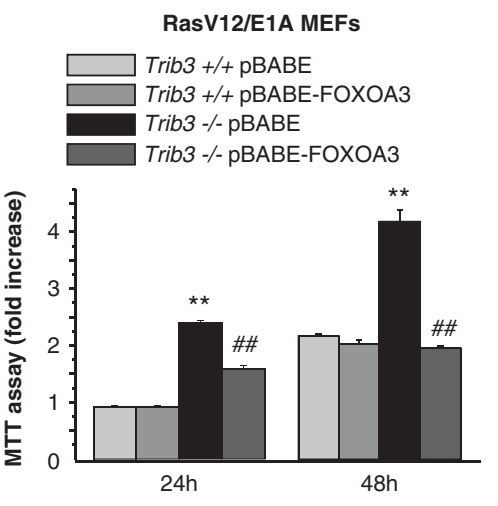

e

Ki67 staining
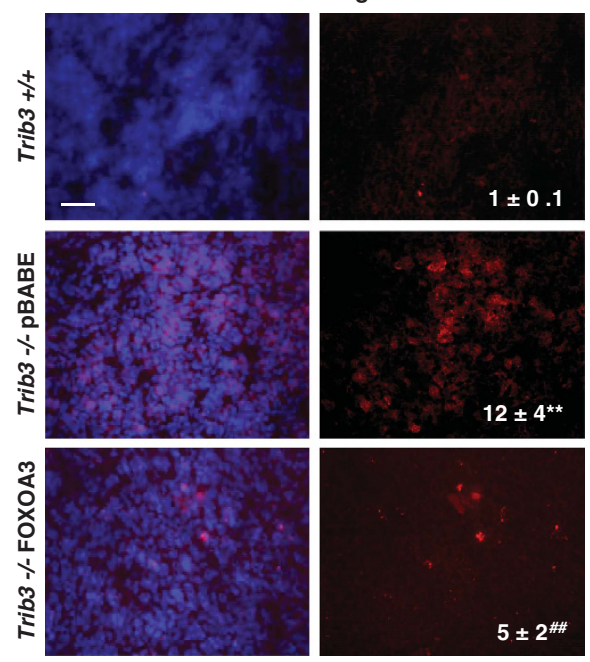

$\mathbf{f}$

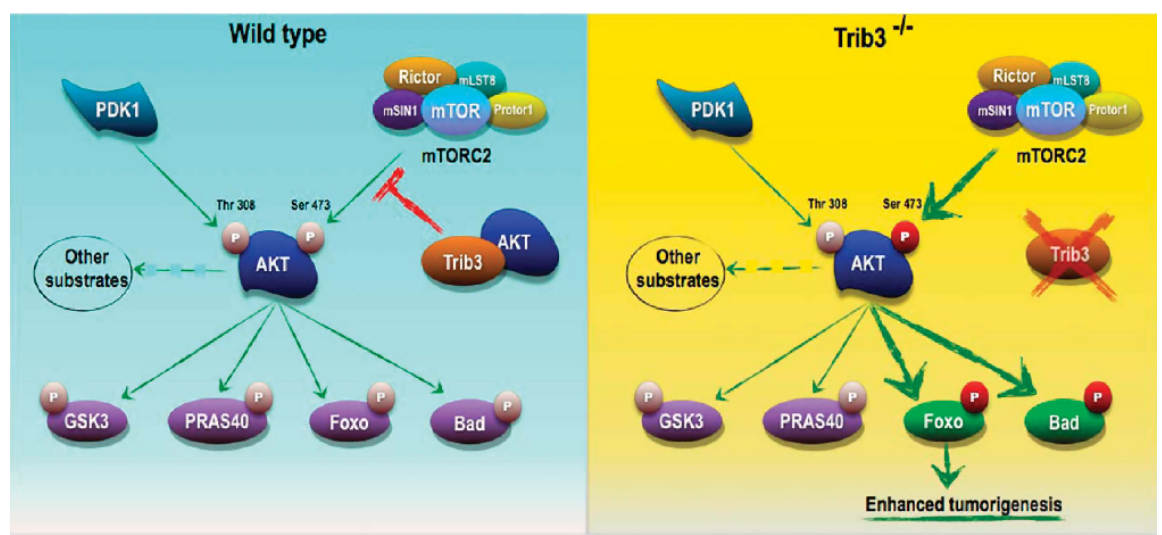


these observations support that $\mathrm{FOXO} 3$ inactivation has a crucial role in the enhanced tumorigenic features of cells in which Trib3 is genetically inactivated. Nevertheless, the expression of the FOXO-A3 mutant affected the rate of tumor growth but did not modify the time to occurrence of tumors derived from Trib3 $^{-1-}$ cells, thereby suggesting that together with the regulation of FOXO activity - TRIB3 may use additional mechanisms to control tumorigenesis. Further research should therefore clarify the role played by FOXO in the regulation of cell survival and proliferation in cells in which TRIB3 has been genetically inhibited. Likewise, future studies should investigate whether the regulation of additional AKT targets (such as BAD) or of other targets of TRIB3 (including, for example, those previously implicated in the control of cell proliferation such as ATF-4, SMAD3 or CtIP ${ }^{3,31-33}$ ) may also contribute to the tumor-suppressor activity of TRIB3.

TRIB3 has been previously shown to interact with AKT, ${ }^{11,15}$ although the precise mechanism by which this interaction regulates the activity of the kinase as well as the physiological consequences of this inhibition had not been completely clarified yet. Data presented here show that TRIB3 regulates the phosphorylation of AKT by the mTORC2 complex. Of potential relevance in this context, Sabatini's group has shown that genetic ablation of the mTORC2 component RICTOR affects the phosphorylation of FOXO but not of GSK3 or TSC2, ${ }^{34}$ thereby suggesting that phosphorylation of AKT in Ser 473 could be sufficient for the kinase to phosphorylate some of its substrates. In line with this observation and with the notion that TRIB3 participates in the regulation of AKT phosphorylation by mTORC2, we found that genetic inactivation of TRIB3 leads to an enhanced phosphorylation of FOXO and BAD but not of GSK3or PRAS 40. Moreover, we recently found that treatment with $\Delta^{9}$-tetrahydrocannabinol (a compound derived from the plant Cannabis sativa that exerts antitumor effects in mouse models of cancer ${ }^{14,15,35}$ ) triggers AKT inhibition via an enhanced interaction of TRIB3 with AKT and a subsequent decrease in the interaction of AKT and TRIB3 with the mTORC2 complex. ${ }^{19}$ Our data also show that Trib3 interacts more strongly with WT AKT, or with AKT mutants in which Thr 308 and Ser 473 have been mutated to Ala, than with AKT mutants in which these two phosphorylatable residues have been mutated to Asp to mimic the negative charge associated with their phosphorylation (author's unpublished observations). Altogether, these observations cogently support that the interaction with TRIB3 negatively regulates $A K T$ by restricting the access of the kinase to the mTORC2 complex (a schematic of the proposed model is shown in Figure 7f). Moreover, our findings also show that TRIB3 may contribute to the regulation of AKT selectivity for some of its substrates. Of note, total Akt levels are increased in Trib3 ${ }^{-/-}$RasV12/E1A-transformed MEFs when compared with their corresponding WT counterparts. Although this effect is much less pronounced than that of TRIB3 genetic inactivation on Akt phosphorylation and does not take place in HepG2 or BT474 cells in which TRIB3 expression was silenced, these observations suggest that TRIB3 may also have a role in the regulation of AKT stability.

Analysis of gene expression profiles of cancer patients from published studies reveals that TRIB3 mRNA levels are downregulated in different tumor types (Supplementary
Tables SII and SIII). Likewise, high TRIB3 protein levels are associated with good prognosis in breast cancer patients. ${ }^{36}$ In addition, TRIB3 is focally deleted in tumors of epithelial origin (tumorscape data set; Supplementary Table SI), thus supporting that loss or downregulation of TRIB3 is an event associated with human cancer. Nevertheless, other studies have found an upregulation of TRIB3 mRNA levels in human cancers. ${ }^{33,37}$ In addition, previous observations by other laboratories indicate that TRIB3 can enhance proliferation and invasiveness of cancer cells in vitro, ${ }^{31-33}$ and a recent study has shown that knockdown of TRIB3 produces a moderate increase in the growth of orthotopic xenografts generated with MDA-231 breast cancer cells. ${ }^{38}$ A plausible explanation for the apparently contradictory results obtained here and in those studies could be the existence of differences in the genetic alterations, the degree of differentiation or other relevant phenotypic characteristics in the cell lines used in the various studies. In support of this idea, and as shown in the present study, silencing of TRIB3 produces heterogeneous effects on the tumorigenic properties of different cancer cell lines as it enhances the growth rate of tumors generated with U87MG cells (data not shown), accelerates the onset of HepG2 cell-derived tumors and hastens both processes in tumors generated with BT474 cells. Thus, although the findings presented here cogently support that genetic inhibition of TRIB3 enhances tumorigenesis in several genetic contexts and, specifically, in the presence of activating mutations of Ras or the deletion of one of the copies of Pten, further research should clarify whether inactivation or enhanced expression of TRIB3 may produce a different outcome in a distinct genetic or cellular context. It is worth noting that it has been reported that TRIB3-null animals show upregulation of TRIB1 and TRIB2 in at least some tissues. ${ }^{39}$ As TRIB2 is associated with induction of acute myelogenous leukemia ${ }^{40,41}$ and TRIB1 with other malignancies, ${ }^{42,43}$ changes in the levels of the other Tribbles isoforms may also contribute to the phenotype observed in Trib3 ${ }^{-1-}$ animals.

In summary, results presented here show that genetic inhibition of TRIB3 increases tumorigenesis in several animal models of cancer and that this effect is due - at least primarily - to the enhanced phosphorylation of AKT by the mTORC2 complex and the subsequent hyperphosphorylation and inactivation of FOXO3. These results may help to set the basis for the development of novel targeted therapies for the management of cancer.

\section{Materials and Methods}

A list of the reagents used, primer sequences, antibodies and other materials, together with additional details of all techniques used in this study can be found in Supplementary Methods.

Generation of Trib3 $^{-/-}$mice. Trib3 ${ }^{+/-}$mice (B6;129S5-Trib3Gt(OST324148)Lex/leg obtained from European Mouse Mutant Archive, EMMA, München, Germany; ID: EM-02346, LEXKO-1947) were back crossed with C57/ BL6 for 10 generations and were then inter-crossed to generate homozygous knockout embryos. This line was developed using the OST324148 OmniBank ES cell line from a sequence tagged gene trap library. ${ }^{44}$ Briefly, the gene trap vector included a two expression cassettes (Supplementary Figure S1a). The first cassette encoded for a splice acceptor site (SA), followed by a fusion protein of beta-galactosidase and neomycin, thereby disrupting transcription of the targeted mRNA. The second cassette encoded for a 'diagnostic marker', followed by a 
splice donor site and was used to determine the site of insertion for the targeting vector by $3^{\prime}$ RACE. Using this gene trap vector, the TRIB3 allele was targeted in the first intron (Supplementary Figure S1b). The sequence shown in Supplementary Figure S1c includes 250 nucleotides on either side of the insertion site, denoted by an asterisk (data kindly provided by Lexicon Pharmaceuticals, The Woodlands, TX, USA). Exons 2-4 encode for the TRIB3 Open Reading Frame (highlighted in red). Insertion of the gene trap vector prevented the expression of the TRIB3 protein, as demonstrated by an anti-TRIB3 western blotting (Supplementary Figure S1e), and did not produce a truncated version of Trib3 as we had previously proposed in a previous work. ${ }^{19}$

Generation of Pten $^{+/-}$Trib3 $^{-/-}$animals. Pten ${ }^{+/-}$mice (C57/B/6/J) were kindly provided by Dr Manuel Serrano (Spanish Cancer Research Center, Madrid, Spain). Characterization of these animals has been previously described ${ }^{45}$ Pten $^{+1-}$ mice were crossed with Trib $^{+1-}$ mice and the progeny inter-crossed. Animals of the different genotypes were killed at 8 months of age, and histopathological analyses of different organs were performed. All procedures involving animals were performed with the approval of the Complutense University Animal Experimentation Committee in compliance with Spanish and European official regulations.

Genotyping. Tail genomic DNA were isolated using standard procedures. Primers used for PCR genotyping were: $5^{\prime}$-CCGCGACGAATGAAAGGTTTA-3'; $5^{\prime}$-AGACTCCGAGAGCTGCTCAGTTAGG-3' for wt (Trib3) allele (483 bp) and $5^{\prime}$-CCGCGACGAATGAAAGGTTAA-3', 5'-AAATGGCGTTACTTAAGCTAGCTTGC-3' for KO (Trib3) allele (381 bp). PCR conditions were 40 cycles $\left(30 \mathrm{~s}\right.$ at $95^{\circ} \mathrm{C}, 30 \mathrm{~s}$ at $65^{\circ} \mathrm{C}$ and $30 \mathrm{~s}$ at $72{ }^{\circ} \mathrm{C}$; supplementary Figure S1). Gentotyping of Pten animals has been previously described. ${ }^{45}$

Skin chemical carcinogenesis model: animals and treatments. As pure C57BL6/J mice are refractory to DMBA/ TPA-induced carcinogenesis, Trib3 $^{-1-}$ animals were bred to the DMBA/TPA-sensitive 129/Ola strain. Heterozygous animals from this progeny were inter-crossed to generate the Trib3 $^{+/+}$and Trib3 $3^{-1-}$ littermates used in this study (129/SvEvBrd_C57BL6/J mixed genetic background). For the two-stage carcinogenesis experiments, tumors were induced on the shaved dorsal skin of WT $(n=15)$ and TRB3 KO $(n=20)$ mice by topical application of a single dose of DMBA ( $32 \mathrm{mg}$ in $200 \mathrm{ml}$ of acetone), followed by twice-weekly applications of TPA (12.5 mg in $200 \mathrm{ml}$ of acetone) for 20 weeks. The number of tumors ( $42 \mathrm{~mm}$ in diameter) was recorded weekly. TPA treatment was ended after 20 weeks, and animals were left untreated until week 32 after DMBA initiation to allow the development of carcinomas. Tumors were collected at different time points and histologically typed as previously described. At the end of the experiments, mice were killed and tumors or skin were collected. Samples were divided into portions that were ${ }^{1}$ frozen in Tissue-Tek (Sakura Finetek Europe, Zoeterwoude, The Netherlands) for immunofluorescence staining, ${ }^{2}$ snap-frozen for protein and RNA extraction or ${ }^{3}$ fixed in $10 \%$ buffered formalin, $\mathrm{pH} 7.2$, for 24-72h. All the tissues were processed by standard histological techniques for the production of $4-\mu \mathrm{m}$ thick sections stained with hematoxylin and eosin. Skin slides were studied and classified according to the International Classification of Rodent Tumours (1993). In four cases of animal death, necropsies were performed on all mice within $24 \mathrm{~h}$ of death, and samples of cutaneous masses, lung, liver, kidney, spleen, stomach, small and large intestine, adrenal and thyroid gland, reproductive tract and brain were collected and processed the same way.

Cell culture. U87MG (human glioma cell line), BT474 (human breast cancer cells) and HEPG2 cells (human hepatocellular carcinoma cell line) were obtained from the American Culture Collection (Rockville, MD, USA). CarC, CarCR, MSC11B9, MSC11A5, PDV and CarB murine skin carcinoma cell lines (see text and Supplementary Methods for further details) were obtained from Miguel Quintanilla. (CarC-R is a less aggressive epithelial cell line isolated from the fusocellular carcinoma cell line CarC, ${ }^{21}$ MSC11B9 (B9) and MSC11A5 (A5) cells were derived, respectively, from the squamous component and the more undifferentiated region of the same carcinoma; ${ }^{20}$ PDV cells were derived from a squamous cell carcinoma; and CarB cells from a spindle cell carcinoma).

Trib3 $^{+1+}$ and Trib3 ${ }^{-1-}$ MEFs were extracted as previously described ${ }^{46}$ and transformed using different retroviral vectors to generate RasV ${ }^{12}$-MEFs, RasV12/

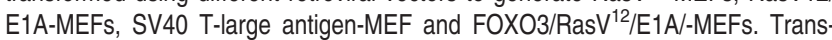
formed/stably transfected MEFs correspond to a polyclonal mix of at least 20 different selected clones). Cells were cultured in DMEM containing 10\% FBS and penicillin/ streptomycin $(5 \mu \mathrm{g} / \mathrm{ml})$. When required, cells were seeded at a density of 5000-10000 cells $/ \mathrm{cm}^{2}$ and transferred to medium containing 0.5\% FBS (except RasV12/E1A-transformed MEFs, which were transferred to media containing $2 \%$ FBS) $18 \mathrm{~h}$ before performing the different treatments.

Genetic knockdown by small interfering RNA. SMARTpool siRNA duplexes (a combination of four siRNAs into a single pool, which guarantees an efficiency of silencing of at least $75 \%$; Dharmacon, Lafayette, CO, USA) were used to transiently silence the expression of the indicated genes. Controls were transfected with a non-targeted control siRNA. Transfection efficiency was $>70 \%$ as monitored with a control fluorescent (red) siRNA (siGLO RISC-Free siRNA, Dharmacon).

Infection with TRB3 shRNA- human lentiviral particles. A pool of concentrated transduction-ready viral particles containing three shRNAs targetspecific (or three shRNA non-targeted control) constructs (Santa Cruz Biotechnology, Heidelberg, Germany) was used to infect human cancer cell lines. Clones of stably silenced (or control shRNA transduced) cells were selected. At least 20 different selected clones were pooled for each of the cell lines generated.

Luciferase assays. Cells were transfected with a luciferase reporter constructs containing different promoters along with a Renilla luciferase expression plasmid (pRL-TK) for normalization.

In vivo generation of tumor xenografts. Tumors were induced by subcutaneous injection (Harlan Interfauna Iberica, Barcelona, Spain) of $4 \times 10^{6}$ cells in PBS supplemented with $0.1 \%$ glucose in nude mice.

Statistics. Except when otherwise stated, statistical analysis was performed by ANOVA with a post-hoc analysis by the Student-Neuman-Keuls test (paired or unpaired). Differences were considered significant when the $P$-value was $<0.05$. Wilcoxon's signed-rank test was used in the analysis of cumulative number of papillomas found in animals subjected to the DMBA/TPA treatment.

\section{Conflict of Interest}

The authors declare no conflict of interest.

Acknowledgements. This work was supported by grants from Spanish Ministry of Economy and Competitiveness (MINECO) and Fondo Europeo de Desarrollo Regional (FEDER) (PS09/01401; PI12/02248, FR2009-0052 and IT2009-0053 to GV), Comunidad de Madrid (S2011/BMD-2308 to MG), Fundación Mutua Madrileña (AP101042012 to GV) and Breast Cancer Campaign (2012NovSP033 to EKT and GV). Purchase of the TRIB3-deficient mice (LEXKO-1947) line was funded by the Wellcome Trust. MS was recipient of a fellowship from Spanish Ministry of Education and Science (MEC), a research formation contract from Comunidad de Madrid and a Postdoctoral Research Contract from Fundación Científica Asociación Española Contra el Cáncer (AECC); $M L$ was sequentially the recipient of a 'Juan de la Cierva' contract, a postdoctoral contract from Spanish Ministry of Education and Science (MEC) and a postdoctoral contract from Comunidad de Madrid. AC is supported by an ERC StG (336343), the Ramón y Cajal award (Spanish Ministry of Education), the Basque Department of Industry, Tourism and Trade (Etortek), Marie Curie Reintegration grant (277043), Movember Global Action Plan, ISCIII (PI10/01484) and the Basque Government of health (2012111086) and education (PI2012-03). EPG was the recipient of a Postdoctoral Research Contract from AECC. We thank Dr Dario Alessi (Dundee University, UK) for his expert scientific and technical support and for kindly providing antibodies for the immnuprecipitation experiments. We also thank other members of our laboratories for their continuous support.

\section{Author contributions}

MS and ML participated in the design of the study, the performance of in vitro and in vivo experiments, the analysis and interpretation of the data and manuscript writings. EGT performed in vitro and in vivo experiments, EPG participated in the design of skin carcinogenesis experiments, analysis and interpretation of the data and critical reading of the manuscript, DD participated in the design and 
performance of in vitro experiments, analysis and interpretation of the data and critical reading of the manuscript, $\mathrm{PZ}$ participated in the performance of in vitro experiments and analysis of the data, JMF and AR performed anatomo-pathological analyses in mice. ZH, DMA and AMA performed bio-informatics analyses, SHT and ILV participated in the performance of in vitro experiments; MQ provided materials and participated in the design of experiments with skin cancer cells; CS, JLI, ND, $M G, S E F, A C$ and EKT participated in the design of the study and critical reading of the manuscript; and GV conceived and coordinated the study and wrote the manuscript.

1. Boudeau J, Miranda-Saavedra D, Barton GJ, Alessi DR. Emerging roles of pseudokinases. Trends Cell Biol 2006; 16: 443-452.

2. Zeqiraj E, van Aalten DM. Pseudokinases-remnants of evolution or key allosteric regulators? Curr Opin Struct Biol 2010; 20: 772-781.

3. Zhang H, Photiou A, Grothey A, Stebbing J, Giamas G. The role of pseudokinases in cancer. Cell Signal 2012; 24: 1173-1184.

4. Grosshans J, Wieschaus E. A genetic link between morphogenesis and cell division during formation of the ventral furrow in Drosophila. Cell 2000; 101: 523-531.

5. Mata J, Curado S, Ephrussi A, Rorth P. Tribbles coordinates mitosis and morphogenesis in Drosophila by regulating string/CDC25 proteolysis. Cell 2000; 101: $511-522$.

6. Rorth $\mathrm{P}$, Szabo K, Texido $\mathrm{G}$. The level of C/EBP protein is critical for cell migration during Drosophila oogenesis and is tightly controlled by regulated degradation. Mol Cell 2000; 6: 23-30.

7. Seher TC, Tribbles Leptin M. a cell-cycle brake that coordinates proliferation and morphogenesis during Drosophila gastrulation. Curr Biol 2000; 10: 623-629.

8. Hegedus Z, Czibula A, Kiss-Toth E. Tribbles: a family of kinase-like proteins with potent signalling regulatory function. Cell Signal 2007; 19: 238-250.

9. Ohoka N, Yoshii S, Hattori T, Onozaki K, Hayashi H. TRB3 a novel ER stress-inducible gene, is induced via ATF4-CHOP pathway and is involved in cell death. EMBO J 2005; 24: 1243-1255.

10. Kiss-Toth E, Bagstaff SM, Sung HY, Jozsa V, Dempsey C, Caunt JC et al. Human tribbles, a protein family controlling mitogen-activated protein kinase cascades. J Biol Chem 2004; 279: 42703-42708.

11. Du K, Herzig S, Kulkarni RN, Montminy M. TRB3: a tribbles homolog that inhibits Akt/PKB activation by insulin in liver. Science 2003; 300: 1574-1577.

12. Koo SH, Satoh H, Herzig S, Lee CH, Hedrick S, Kulkarni R et al. PGC-1 promotes insulin resistance in liver through PPAR-alpha-dependent induction of TRB-3. Nat Med 2004; 10: 530-534.

13. Carracedo A, Gironella M, Lorente M, Garcia S, Guzman M, Velasco G et al. Cannabinoids induce apoptosis of pancreatic tumor cells via endoplasmic reticulum stress-related genes. Cancer Res 2006; 66: 6748-6755.

14. Carracedo A, Lorente M, Egia A, Blazquez C, Garcia S, Giroux V et al. The stressregulated protein p8 mediates cannabinoid-induced apoptosis of tumor cells. Cancer Cell 2006; 9: 301-312.

15. Salazar M, Carracedo A, Salanueva IJ, Hernandez-Tiedra S, Lorente M, Egia A et al. Cannabinoid action induces autophagy-mediated cell death through stimulation of ER stress in human glioma cells. J Clin Invest 2009; 119: 1359-1372.

16. Nicoletti-Carvalho JE, Nogueira TC, Gorjao R, Bromati CR, Yamanaka TS, Boschero AC et al. UPR-mediated TRIB3 expression correlates with reduced AKT phosphorylation and inability of interleukin 6 to overcome palmitate-induced apoptosis in RINm5F cells. J Endocrinol 2010; 206: 183-193.

17. Vara D, Salazar M, Olea-Herrero N, Guzman M, Velasco G, Diaz-Laviada I. Anti-tumoral action of cannabinoids on hepatocellular carcinoma: role of AMPK-dependent activation of autophagy. Cell Death Differ 2011; 18: 1099-1111.

18. Zhang J, Wen HJ, Guo ZM, Zeng MS, Li MZ, Jiang YE et al. TRB3 overexpression due to endoplasmic reticulum stress inhibits AKT kinase activation of tongue squamous cell carcinoma. Oral Oncol 2011; 47: 934-939.

19. Salazar M, Lorente M, Garcia-Taboada E, Hernandez-Tiedra S, Davila D, Francis SE et al. The pseudokinase tribbles homologue-3 plays a crucial role in cannabinoid anticancer action. Biochim Biophys Acta 2013; 1831: 1573-1578.

20. Burns PA, Kemp CJ, Gannon JV, Lane DP, Bremner R, Balmain A. Loss of heterozygosity and mutational alterations of the p53 gene in skin tumours of interspecific hybrid mice. Oncogene 1991; 6: 2363-2369.
21. Pons M, Cigudosa JC, Rodriguez-Perales S, Bella JL, Gonzalez C, Gamallo C et al. Chromosomal instability and phenotypic plasticity during the squamous-spindle carcinoma transition: association of a specific T(14;15) with malignant progression. Oncogene 2005; 24: 7608-7618.

22. Abel EL, Angel JM, Kiguchi K, DiGiovanni J. Multi-stage chemical carcinogenesis in mouse skin: fundamentals and applications. Nat Protoc 2009; 4: 1350-1362.

23. Pearce LR, Komander D, Alessi DR. The nuts and bolts of AGC protein kinases. Nat Rev Mol Cell Biol 2009; 11: 9-22.

24. Zoncu R, Efeyan A, Sabatini DM. mTOR: from growth signal integration to cancer, diabetes and ageing. Nat Rev Mol Cell Biol 2010; 12: 21-35.

25. Salmena L, Carracedo A, Pandolfi PP. Tenets of PTEN tumor suppression. Cell 2008; 133 : 403-414.

26. Nardella C, Carracedo A, Salmena L, Pandolfi PP. Faithfull modeling of PTEN loss driven diseases in the mouse. Curr Top Microbiol Immunol 2011; 347: 135-168.

27. Hollander MC, Blumenthal GM, Dennis PA. PTEN loss in the continuum of common cancers, rare syndromes and mouse models. Nat Rev Cancer 2011; 11: 289-301.

28. Dansen TB, Burgering BM. Unravelling the tumor-suppressive functions of FOXO proteins. Trends Cell Biol 2008; 18: 421-429.

29. Biggs WH 3rd, Meisenhelder J, Hunter T, Cavenee WK, Arden KC. Protein kinase B/Aktmediated phosphorylation promotes nuclear exclusion of the winged helix transcription factor FKHR1. Proc Natl Acad Sci USA 1999; 96: 7421-7426.

30. Brunet A, Bonni A, Zigmond MJ, Lin MZ, Juo P, Hu LS et al. Akt promotes cell survival by phosphorylating and inhibiting a Forkhead transcription factor. Cell 1999; 96: 857-868.

31. Bowers AJ, Scully S, Boylan JF. SKIP3 a novel Drosophila tribbles ortholog, is overexpressed in human tumors and is regulated by hypoxia. Oncogene 2003; 22: 2823-2835.

32. Hua F, Mu R, Liu J, Xue J, Wang Z, Lin H et al. TRB3 interacts with SMAD3 promoting tumor cell migration and invasion. J Cell Sci 2011; 124(Pt 19): 3235-3246.

33. Xu J, Lv S, Qin Y, Shu F, Xu Y, Chen J et al. TRB3 interacts with CtIP and is overexpressed in certain cancers. Biochim Biophys Acta 2007; 1770: 273-278.

34. Guertin DA, Stevens DM, Thoreen CC, Burds AA, Kalaany NY, Moffat $J$ et al. Ablation in mice of the mTORC components raptor, rictor, or $\mathrm{mLST} 8$ reveals that mTORC2 is required for signaling to Akt-FOXO and PKCalpha, but not S6K1. Dev Cell 2006; 11: 859-871.

35. Velasco G, Sanchez C, Guzman M. Towards the use of cannabinoids as antitumour agents. Nat Rev Cancer 2012; 12: 436-444.

36. Wennemers M, Bussink J, Grebenchtchikov N, Sweep FC, Span PN. TRIB3 protein denotes a good prognosis in breast cancer patients and is associated with hypoxia sensitivity. Radiother Oncol 2011; 101: 198-202.

37. Wennemers M, Bussink J, Scheijen B, Nagtegaal ID, van Laarhoven HW, Raleigh JA et al. Tribbles homolog 3 denotes a poor prognosis in breast cancer and is involved in hypoxia response. Breast Cancer Res 2011; 13: R82.

38. Izrailit J, Berman HK, Datti A, Wrana JL, Reedijk M. High throughput kinase inhibitor screens reveal TRB3 and MAPK-ERK/TGFbeta pathways as fundamental Notch regulators in breast cancer. Proc Natl Acad Sci USA 2013; 110: 1714-1719.

39. Zareen N, Biswas SC, Greene LA. A feed-forward loop involving Trib3, Akt and FoxO mediates death of NGF-deprived neurons. Cell Death Differ 2013; 20: 1719-1730.

40. Rishi L, Hannon M, Salome M, Hasemann M, Frank AK, Campos J et al. Regulation of Trib2 by an E2F1-C/EBPalpha feedback loop in AML cell proliferation. Blood 2014; 123: 2389-2400.

41. Zanella F, Renner O, Garcia B, Callejas S, Dopazo A, Peregrina S et al. Human TRIB2 is a repressor of FOXO that contributes to the malignant phenotype of melanoma cells. Oncogene 2010; 29: 2973-2982.

42. Jin G, Yamazaki Y, Takuwa M, Takahara T, Kaneko K, Kuwata T et al. Trib1 and Evi1 cooperate with Hoxa and Meis1 in myeloid leukemogenesis. Blood 2007; 109: 3998-4005.

43. Mashima T, Soma-Nagae T, Migita T, Kinoshita R, Iwamoto A, Yuasa T et al. TRIB1 supports prostate tumorigenesis and tumor-propagating cell survival by regulation of endoplasmic reticulum chaperone expression. Cancer Res 2014; e-pub ahead of print 24 June 2014.

44. Zambrowicz BP, Abuin A, Ramirez-Solis R, Richter LJ, Piggott J, BeltrandelRio $\mathrm{H}$ et al. Wnk1 kinase deficiency lowers blood pressure in mice: a gene-trap screen to identify potential targets for therapeutic intervention. Proc Natl Acad Sci USA 2003; 100: 14109-14114.

45. Di Cristofano A, Pesce B, Cordon-Cardo C, Pandolfi PP. Pten is essential for embryonic development and tumour suppression. Nat Genet 1998; 19: 348-355.

46. Salazar M, Hernandez-Tiedra S, Torres S, Lorente M, Guzman M, Velasco G. Detecting autophagy in response to ER stress signals in cancer. Methods Enzymol 2011; 489: 297-317. 